Review

\title{
Exploiting the Biosynthetic Potential of Type III Polyketide Synthases
}

\author{
Yan Ping Lim ${ }^{1,2}$, Maybelle K. Go ${ }^{1,2}$ and Wen Shan Yew ${ }^{1,2, *}$ \\ 1 Department of Biochemistry, Yong Loo Lin School of Medicine, National University of Singapore, \\ 8 Medical Drive, Singapore 117597, Singapore; bchlimy@nus.edu.sg (Y.P.L.); bchmdkg@nus.edu.sg (M.K.G.) \\ 2 NUS Synthetic Biology for Clinical and Technological Innovation, Centre for Life Sciences, \\ National University of Singapore, 28 Medical Drive, Singapore 117456, Singapore \\ * Correspondence: wen_shan_yew@nuhs.edu.sg; Tel.: +65-6516-8624
}

Academic Editor: Kira Weissman

Received: 20 May 2016; Accepted: 17 June 2016; Published: 22 June 2016

\begin{abstract}
Polyketides are structurally and functionally diverse secondary metabolites that are biosynthesized by polyketide synthases (PKSs) using acyl-CoA precursors. Recent studies in the engineering and structural characterization of PKSs have facilitated the use of target enzymes as biocatalysts to produce novel functionally optimized polyketides. These compounds may serve as potential drug leads. This review summarizes the insights gained from research on type III PKSs, from the discovery of chalcone synthase in plants to novel PKSs in bacteria and fungi. To date, at least 15 families of type III PKSs have been characterized, highlighting the utility of PKSs in the development of natural product libraries for therapeutic development.
\end{abstract}

Keywords: chalcone synthase; stilbene synthase; polyketide; precursor-directed combinatorial biosynthesis; synthetic enzymology

\section{Introduction}

Substantial focus has been awarded to polyketide research since its initial discovery about a century ago [1]. Polyketides are structurally and functionally diverse secondary metabolites produced in bacteria, fungi, and plants. Many of these bioactive natural products have significant medical or agricultural applications [2], such as rapamycin (a macrolide immunosuppressant) [3], lovastatin (used for the treatment of hypercholesterolemia) [4], actinorhodin (an antibiotic) [5], and resveratrol (reported anti-ageing properties in model organisms) [6] (Figure 1). This highlights the vital role polyketides and their derivatives can play in the development of novel therapeutics [7]. Although chemical synthesis can be used to produce polyketide analogues [8], the chemical and structural complexity of polyketides and their derivatives prevent routine production by this route.

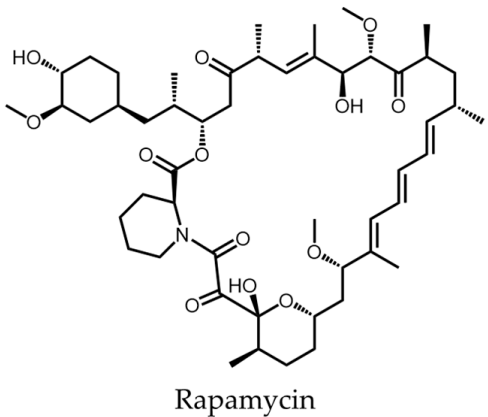

(Modular Type I PKS)

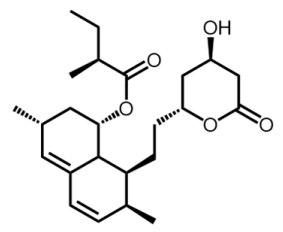

Lovastatin

(Iterative Type I PKS)

Figure 1. Cont. 


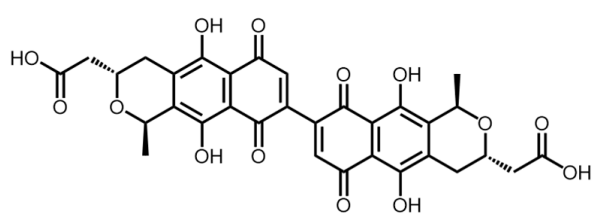

Actinorhodin

(Type II PKS)<smiles>Oc1ccc(/C=C/c2cc(O)cc(O)c2)cc1</smiles>

Resveratrol

(Type III PKS)

Figure 1. Examples of polyketides synthesized by the different classes of PKSs.

Insights into biosynthetic mechanisms of polyketides only achieved considerable progress from 1953 when Birch and Donovan hypothesized a potential biosynthetic pathway similar to that of fatty acids [9]. Further advancements in the polyketide field led to the consensus that polyketides are typically biosynthesized through successive decarboxylative condensations of coenzyme A (CoA)-derived units, into a complex polycyclic multi-carbon compound containing keto or hydroxyl groups [10]. Downstream modifying enzymes are then used to obtain flavonoids and other bioactive substances. Recent studies in the engineering and structural characterization of polyketide synthases (PKSs) have facilitated the use of target enzymes as biocatalysts to produce novel functionally optimized polyketides, which can serve as potential drug leads [11,12].

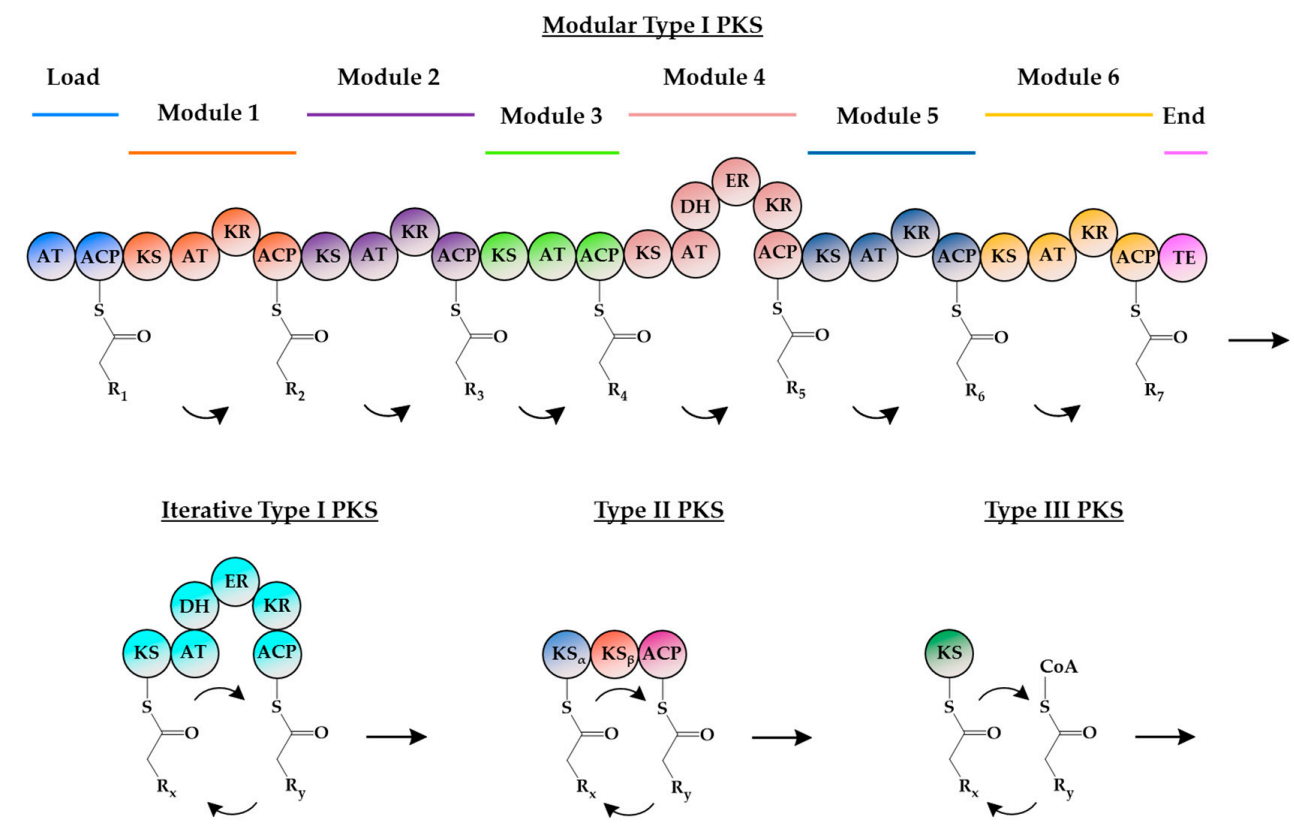

Figure 2. Domain organization of the different PKSs. Putative domains are represented by circles. In modular type I PKSs, functional domains are organized into several modules, with each module being responsible for a single decarboxylative condensation step in polyketide formation. For iterative type I PKSs, the functional domains are clustered in a single module, and each domain is used repeatedly during polyketide synthesis. Type II PKSs are dissociable multi-enzyme complexes, with each protein bearing a single and independent catalytic domain that is used iteratively during polyketide formation. Reactions by type III PKSs are also iterative but do not require an ACP for the attachment of the growing polyketide chain. AT: Acyltransferase; ACP: Acyl carrier protein; KS: Ketosynthase; KR: Ketoreductase; DH: Dehydratase; ER: Enoyl reductase; TE: Thioesterase; CoA: Coenzyme A.

Generally, PKSs are grouped into three classes: type I, II, or III PKS (Figure 2) [2]. Type I PKSs are large multi-domain polypeptides which can function in either a modular or iterative manner, shuttling substrates between functional domains via acyl carrier proteins (ACPs). In modular type I PKSs, functional domains are organized into several modules, with each module being responsible for a single decarboxylative condensation step in the formation of the polyketide $[2,13]$. Conversely for 
iterative type I PKSs, the functional domains are clustered in a single module; hence, each domain is used repeatedly during polyketide synthesis [14]. Type II PKSs are dissociable multi-enzyme complexes, with each protein in the complex bearing a single and independent catalytic domain that is used iteratively during polyketide formation [13]. Type III PKSs are homodimeric enzymes and are structurally simpler and mechanistically different from type I and II PKSs. The relative simplicity, versatility, and unusually broad substrate specificity of type III PKSs make them ideal candidates for the engineering of biocatalysts, and in doing so, access to bioactive polyketide compound libraries can be readily made available [15].

This review highlights recent progress in the understanding of type III PKSs (in particular with regards to the diversity of naturally occurring reactions and products discovered), and summarizes strategies for exploiting the biosynthetic potential of type III PKSs. Approaches based on random mutagenesis or site-directed mutagenesis of active site cavities to engineer the substrate specificity and activity of type III PKSs will also be discussed. These studies serve as a primer towards the development of novel and valuable polyketides with therapeutic potential.

\section{Insights into Type III PKSs}

Type III PKSs were previously only found in plants, but in the past 15 years, more bacterial type III PKSs were characterized [16-18]; fungal type III PKSs were only recently discovered [19-21]. Unlike the type I and II PKSs, type III PKSs generally utilize CoA thioesters as substrates and do not entail the involvement of ACP domains. Furthermore, type III PKSs are able to accomplish an entire series of decarboxylative condensations and cyclization reactions in a single active site [2]. By having a simple architecture, it enables type III PKSs to be amenable to in vitro manipulation and examination.

Although type III PKSs have been studied since the 1980s [22], structural information, on how a single active site can direct the chemistry of several decarboxylative condensations and subsequent cyclization of the polyketide, was only made known after the determination of the crystal structure of chalcone synthase (CHS) from Medicago sativa (commonly known as alfalfa) [23]. This was the first crystal structure of a PKS and it facilitated a framework for engineering type III PKSs to generate novel polyketides.

\subsection{Chalcone Synthases and the Basis of Polyketide Synthesis}

CHSs are the most well studied type III PKSs as they are ubiquitous in higher plants and essential for plant metabolism and defense [15]. Based on the structure of CHS from alfalfa (PDB ID: 1CGK), it was found that the symmetric dimer encapsulates a CoA-binding tunnel, a coumaroyl-binding pocket, and a cyclization pocket in order to facilitate chalcone biosynthesis [23]. Together with the conserved Cys164-His303-Asn336 catalytic triad, and the 'gatekeeper' Phe215 which is hypothesized to aid in the orientation of substrates during polyketide chain elongation, these connected cavities constitute the active site architecture of a typical type III PKS [24,25]. Chalcone synthesis by CHS is initiated by the loading of $p$-coumaroyl-CoA (1) onto the sulfhydryl group at the active site cysteine residue. The reaction then proceeds on with three iterative decarboxylative condensations of the extender substrate, malonyl-CoA (2), with the cysteine-bound starter unit, aided by the active site histidine and asparagine residues. After chain extension, the linear tetraketide, $p$-coumaroyl triacetyl thioester (4), undergoes an intramolecular C6 to C1 Claisen condensation and cyclizes into chalcone 7, which undergoes a further Michael-type ring closure to give rise to naringenin (8) (Scheme 1) [24].

Numerous type III PKSs have been identified till date, and the range of reactions and product classes discovered is due to differences in intramolecular cyclizations of the linear polyketide intermediate, the number of polyketide extension steps involved, and the choice of starter and extender CoAs used [26]. The diverse polyketide scaffolds catalyzed by type III PKSs can be classified into chalcone, stilbene, pyrone, chromone, stilbenecarboxylate, bibenzyl, benzalacetone, curcuminoid, benzophenone, biphenyl, acridone, quinolone, phloroglucinol, resorcinol, and naphthalene families [24,27], respectively. By characterizing various type III PKSs, we can understand reaction mechanisms better and thus, direct the generation of unnatural polyketides using synthetic enzymology [28]. 


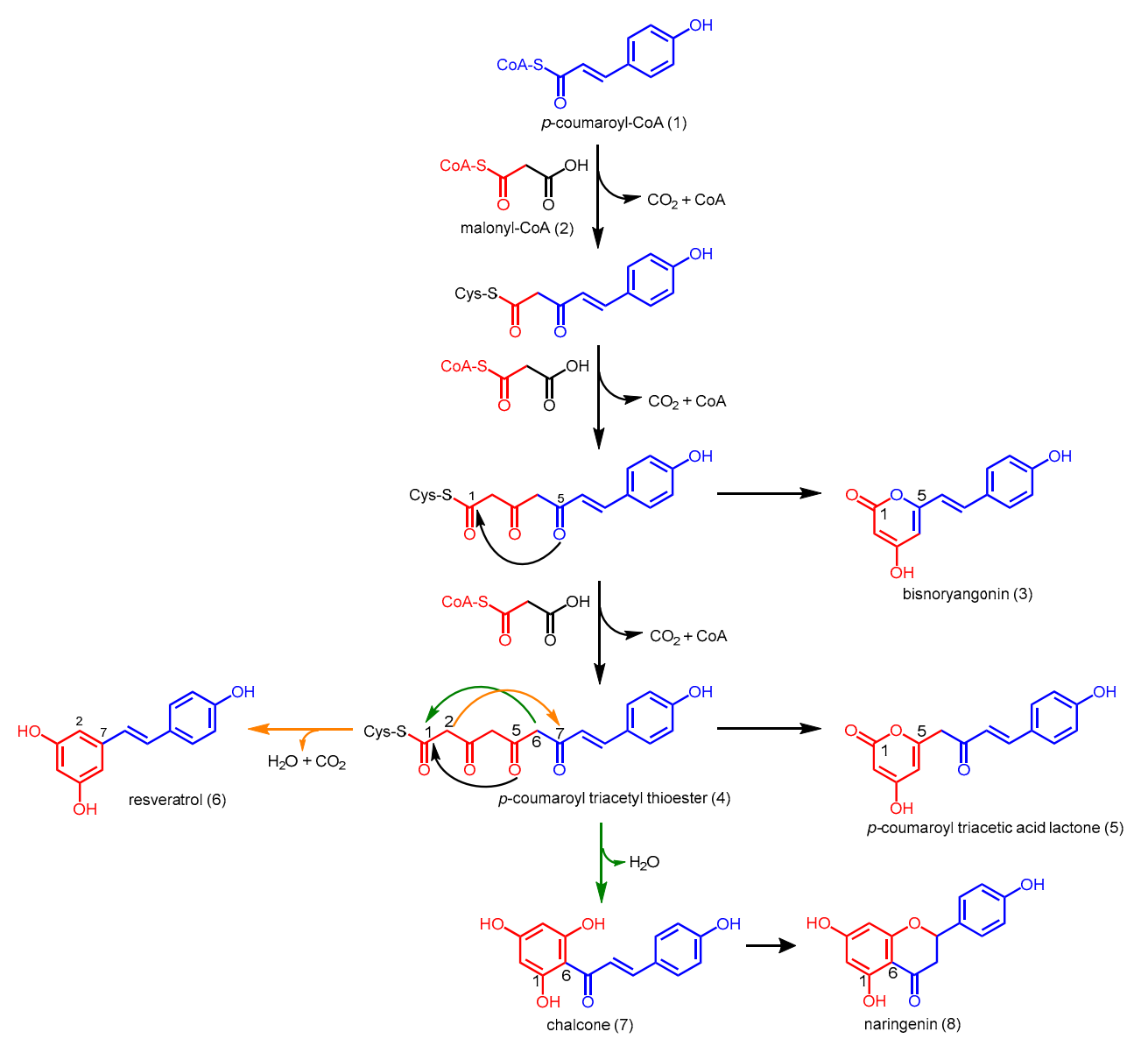

Scheme 1. Chalcone and naringenin synthesis catalyzed by M. sativa CHS or Pinus sylvestris STS.

\subsection{Stilbene Synthases and the Functional Divergence of Type III PKSs}

Stilbene synthases (STSs) are found in only a small subset of unrelated plants, unlike CHSs, which are ubiquitous. Phylogenetic studies show that no ancestral STS gene was found, and STSs are likely to have evolved from CHSs independently on several occasions [29]. STSs and CHSs are structurally related and share at least $65 \%$ amino acid sequence identity [30]. These enzymes follow a similar overall reaction mechanism which involves initiation, followed by the iterative decarboxylative condensation of three units of malonyl-CoA to a starter unit, and cyclization of the linear tetraketide intermediate. Slight variations in active site residues enable STSs to catalyze an intramolecular C2 to C7 aldol condensation, followed by decarboxylation and dehydration to generate a stilbene scaffold instead of a chalcone backbone (Scheme 1) [26].

Although it has been postulated that divergence was due to some steric changes of the active site cavity affecting the orientation of the linear tetraketide intermediate, the questions of how $\mathrm{CHS}$ and STS can produce resveratrol (3,5, $4^{\prime}$-trihydroxystilbene, 6$)$ and naringenin (8) respectively as minor products (Scheme 1) [30], and how decarboxylation occurs in STSs, remain unanswered. Previous homology modeling studies have shown no substantial structural or chemical differences in the STS active site, compared to CHS, and no obvious STS or CHS consensus sequence was identified [26,29]. With the report of the first STS crystal structure from Pinus sylvestris (Scots pine) in 2004 (PDB ID: $1 \mathrm{U} 0 \mathrm{U})$, the mechanism of stilbene synthesis was finally resolved $[26,27]$.

Structure-based mutagenic transformation of alfalfa CHS into STS established that STS's alternative cyclization specificity is due to a conformational backbone change in a short, concealed loop between residues 132 and 137 in alfalfa CHS, that spans the dimer interface in the active site. Several amino acid substitutions at buried sites adjacent to this loop bring about a slight displacement of 
Thr132, a residue that is conserved in both CHS and STS. Consequently, the side chain hydroxyl group of Thr132 is brought within the hydrogen bonding distance of a Ser338-stabilized water molecule adjacent to the catalytic cysteine, and a different active site hydrogen bond network involving Glu192-Thr132- $\mathrm{H}_{2} \mathrm{O}-\mathrm{Ser} 338$ forms in STS (Figure 3) [24,26,31]. The nucleophilic water activated by this "aldol-switch" hydrogen bond network subsequently participates in the cleavage of the thioester bond of the cysteine-bound linear tetraketide intermediate. With the absence of the ester bond at $\mathrm{C} 1$, the intermediate is able to undergo a $\mathrm{C} 2$ to $\mathrm{C} 7$ aldol condensation and ultimately generates a stilbene scaffold. Intriguingly, when an ester bond is present at $\mathrm{C} 1$, a C6 to $\mathrm{C} 1 \mathrm{Claisen}$ condensation prevails [26,32]. Hence in the case of CHS and STS, the functional divergence is determined by electronic factors rather than steric effects of the active site cavity. This also explains the cross-reactivity of CHS and STS previously identified by Yamaguchi and co-workers [30]. Nevertheless, the amino acid substitutions which result in a displacement of Thr132 are varied in different STSs, probably due to gene duplication and convergent evolution. Further biochemical and structural studies on similar proteins will be required to delineate the molecular basis in greater detail [31].

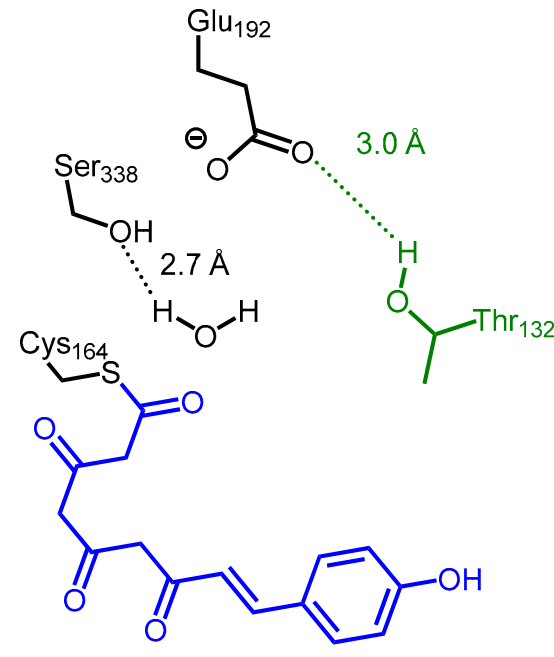

CHS-like active site

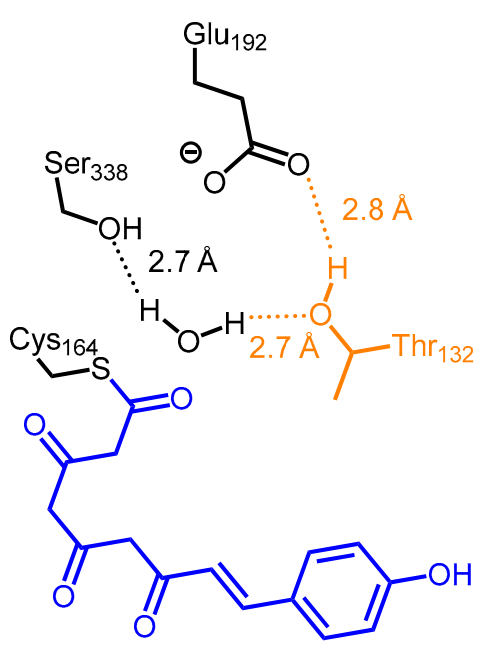

STS-like active site

Figure 3. The hydrogen bond network involving Glu192, Thr132, Ser338, and $\mathrm{H}_{2} \mathrm{O}$. Differences in this network switches the chemistry of the active site of $M$. sativa CHS or P. sylvestris STS between chalcone and stilbene synthesis.

\subsection{Truncation Products and Chain Length Specificity}

Some CHSs and STSs are promiscuous in terms of the number of elongation steps, and polyketide intermediates can become derailed without forming the final chalcone or stilbene product respectively [24]. These intermediates usually undergo an intramolecular C5-oxygen to C1 lactonization to give rise to heterocyclic truncation products. In the case of alfalfa CHS and Scots pine STS, derailed triketide and tetraketide intermediates form bisnoryangonin (3) and coumaroyl triacetic acid lactone (CTAL, 5), respectively (Scheme 1). Although lactone derailment products can form spontaneously from linear polyketide intermediates in acidic solutions [24,30], a 2-pyrone synthase (2-PS) isolated from Gerbera hybrida (daisy) was identified to be able to control the final length of the polyketide and thus, was the first type III PKS to have a different product profile from CHS and STS [33,34].

Despite 2-PS and CHS sharing at least $70 \%$ amino acid sequence identity and identical catalytic residues, 2-PS catalyzes the condensation of an acetyl-CoA starter molecule with only two units of malonyl-CoA to form a triketide, which further undergoes lactonization to generate 6-methyl-4-hydroxy-2-pyrone (9, Figure 4) [34,35]. A closer inspection of the crystal structure of 2-PS (PDB ID: 1QLV) revealed that the active site of 2-PS is only approximately one third of the volume seen 
in CHS, owing to the greater steric bulk of three active site residues [24]. Intriguingly, the triple mutant T197L/G256L/S338I of alfalfa CHS was found to be functionally identical to 2-PS. The increased steric bulk at positions 197 and 338 occludes the coumaroyl-binding pocket, thus excluding big starter units such as phenylpropanoid CoA thioesters. Substitution of Gly256 in CHS with Leu261 in 2-PS reduces the size of the cyclization pocket of the active site, therefore limiting the number of chain extensions of the polyketide [24,35].

(A)

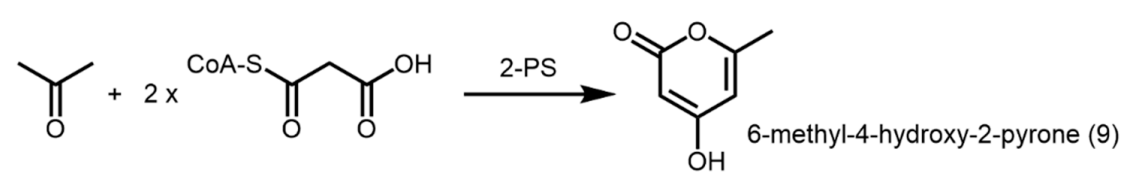

(B)

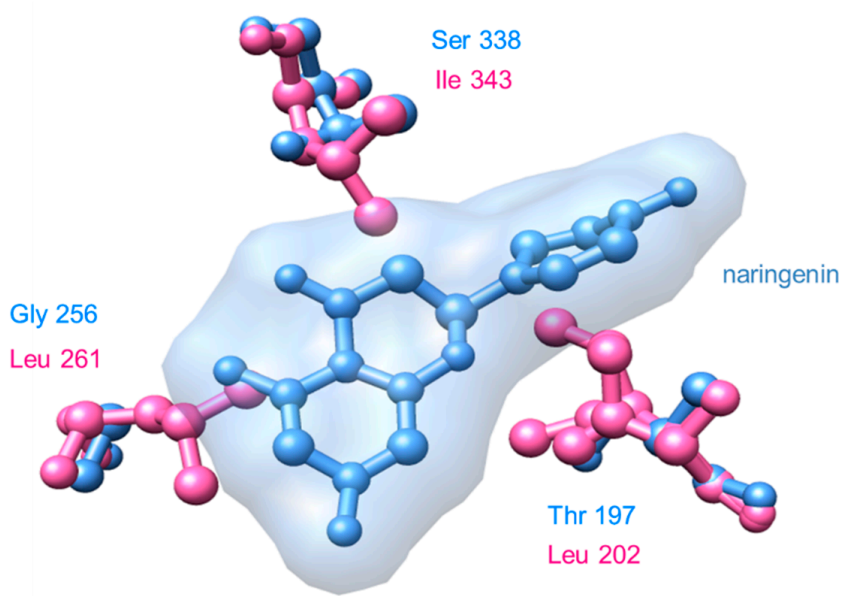

Figure 4. Comparing residues lining the active site of G. hybrida 2-PS and M. sativa CHS. (A) 2-PS catalyzes the condensation of acetyl-CoA with two units of malonyl-CoA to form 6-methyl-4-hydroxy-2-pyrone; (B) the structures of 2-PS (pink, PDB ID: 1QLV) and CHS (blue, PDB ID: 1CGK) were aligned to compare the active site cavity between the two structures. The presence of the three bulkier residues in 2-PS (L202, L261, and I343) show that a tetraketide such as naringenin will not fit inside the active site. Thus, only a smaller polyketide, such as 6-methyl-4-hydroxy-2-pyrone, may be formed.

Conversely, modifications in the active site cavity may also enable the formation of longer polyketides. Aloesone synthase (ALS) from Rheum palmatum (rhubarb) catalyzes six successive decarboxylative condensations of malonyl-CoA with an acetyl-CoA starter molecule, followed by an intramolecular $\mathrm{C} 8$ to $\mathrm{C} 13$ aldol condensation and decarboxylation to generate a heptaketide aloesone (2-acetonyl-7-hydroxy-5-methylchromone) (10) (Scheme 2). As with other plant type III PKSs, ALS shares more than $60 \%$ identity with alfalfa CHS, and maintains the Cys-His-Asn catalytic triad at positions 164, 303, and 336 respectively (numbering in alfalfa CHS) [36,37]. Interestingly, ALS lacks the conserved CHS residues at positions 197,256, and 338, thus affecting the shape and volume of the initiation and elongation cavity of the active site. In fact, these three residues are also sterically altered in the functionally divergent G. hybrida 2-PS (T197L/G256L/S338I) [35]. Further research on novel type III PKSs in the medicinal plant Aloe arborescens (aloe) also revealed similar replacements in the pentaketide chromone synthase (PCS) (T197M/G256L/S338V) [38], heptaketide synthase (PKS3) (T197A/G256L/S338I) [39], and octaketide synthase (OKS) (T197G/G256L/S338V) [40]. These enzymes also catalyze successive decarboxylative condensations of malonyl-CoA with an acetyl-CoA starter molecule to form 5,7-dihydroxy-2-methylchromone (11) (pentaketide), aloesone, and SEK4 (12)/SEK4b (13) (octaketide) respectively (Scheme 2). 2-PS, PCS, PKS3, ALS, and OKS also generate their respective products by an initial decarboxylation of malonyl-CoA to produce acetyl-CoA, which is then utilized as the starter unit. 
(A)

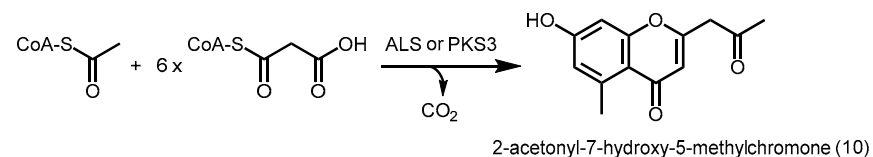

(B)

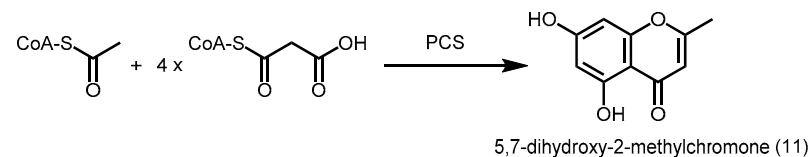

(C)
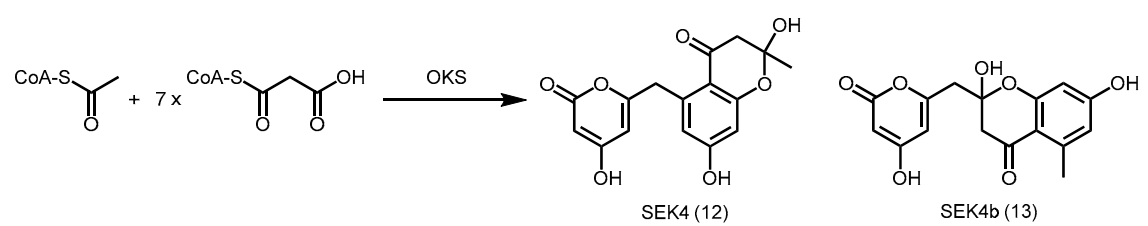

Scheme 2. Polyketide synthesis catalyzed by R. palmatum ALS, A. arborescens PKS3, A. arborescens PCS, and A. arborescens OKS. (A) ALS or PKS3 catalyzes the condensation of one unit of acetyl-CoA and six units of malonyl-CoA, generating 2-acetonyl-7-hydroxy-5-methylchromone; (B) PCS catalyzes the condensation of one unit of acetyl-CoA and four units of malonyl-CoA, generating 5,7-dihydroxy-2-methylchromone; (C) OKS catalyzes the condensation of one unit of acetyl-CoA and seven units of malonyl-CoA to form SEK4 and SEK4b. Acetyl-CoA is biosynthesized from decarboxylation of malonyl-CoA.

Based on homology modeling with CHS, it was predicted that the active site architecture of ALS involves a 'horizontally restricting' bulky replacement at residue position 256 from glycine to leucine and a 'downward expanding' substitution at residue position 197 from threonine to alanine when compared to CHS [37]. Figure 5 shows a schematic of the effect of the residues at positions 197, 256, and 338 for R. palmatum ALS, G. hybrida 2-PS, A. arborescens PCS, A. arborescens PKS3, A. arborescens OKS, and M. sativa CHS. The residue at position 256 is found to line the initiation/elongation cavity and occupies an important site for the loading of the starter molecule, hence facilitating the use of a smaller acetyl-CoA rather than the bulky $p$-coumaroyl-CoA. The reduced steric bulk due to the threonine to alanine substitution at residue position 197 in ALS unlocks a buried pocket, thus expanding a putative cyclization cavity. Site-directed mutagenesis of ALS revealed that changing any amino acid at these positions into the CHS conserved residues (A197T, L256G, and T338S) completely prevented the formation of the heptaketide. In particular, the ALS L256G mutant was able to accept $p$-coumaroyl CoA as a starter unit to generate CTAL, thus suggesting that Gly256 controls starter unit selectivity. Further, the ALS A197T mutant was only able to form a pentaketide 2,7-dihydroxy-5-methylchromone after a C4 to C9 aldol condensation, which is a regioiosmer of the 5,7-dihydroxy-2-methylchromone (11) synthesized by PCS (C6 to C1 Claisen condensation) (Figure 6). Therefore, Thr197 is likely to control polyketide chain length as it is positioned at the entrance of the buried pocket. Thr338 is thought to guide the linear polyketide intermediate, which is bound to the nearby catalytic Cys164, to extend into the buried pocket, hence enabling the formation of the heptaketide.

Interestingly, further mutation of Ala197 in ALS to less bulky Gly197 in OKS (numbering in alfalfa CHS) resulted in the synthesis of SEK4 (12) and SEK4b (13, Scheme 2), the longest polyketides known to be produced by a wild-type type III PKS [37]. Mutagenesis of Met197 in PCS to Gly197 also led to the synthesis of octaketides. Moreover, the cyclization chemistry was altered from a C6 to C1 Claisen condensation of a pentaketide intermediate, to a C12 to C7 and C10 to C15 aldol condensation of an octaketide intermediate to form SEK4 and SEK4b, respectively [38]. Hence, Met197 (and Thr197 in alfalfa CHS) could be important for facilitating Claisen condensation reactions as well [24].

All in all, these findings establish that altering the size and shape of the active site cavity via site-directed mutagenesis contributes to specificity for starter molecule choice, chain elongation, and cyclization chemistry, leading to functional diversity among type III PKSs. This suggests potentially different strategies for the engineered biosynthesis of novel pharmaceutically relevant polyketides. 

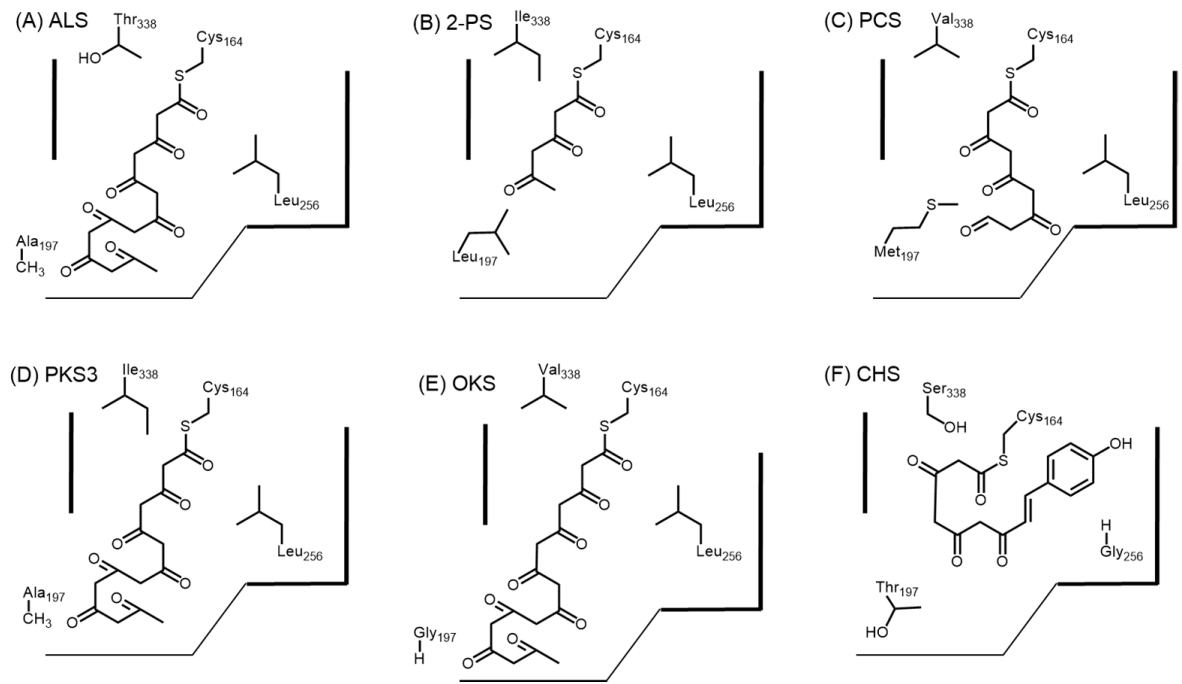

Figure 5. Schematic representation of the active sites of (A) R. Palmatum ALS; (B) G. hybrida 2-PS; (C) A. arborescens PCS; (D) A. arborescens PKS3; (E) A. arborescens OKS; and (F) M. sativa CHS. The residue at position 256 controls the starter unit selectivity. Bulky residues such as leucine prevents the entrance of starter acyl-CoAs such as $p$-coumaroyl CoA. The residue at position 197 controls the gate to an additional buried pocket that extends to the "floor" of the active site. Small residues such as alanine or glycine allows chain extension to form octaketides. Residue at position 338 located at the proximity of the catalytic Cys164 is at the "ceiling" of the active site cavity and this guides the growing polyketide chain to extend into the buried pocket near residue position 197. The numbering for all enzymes are based in M. sativa CHS.

(A)

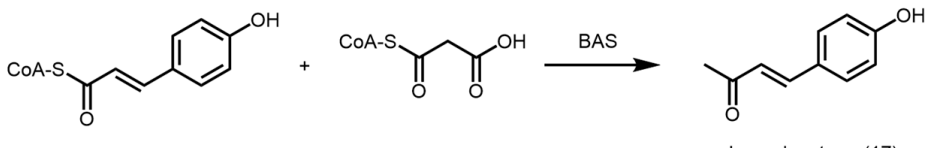

(B) BAS<smiles>CCCCCCCCCCC(=O)CC=Cc1ccc(O)cc1</smiles>

$\mathrm{CHS}$

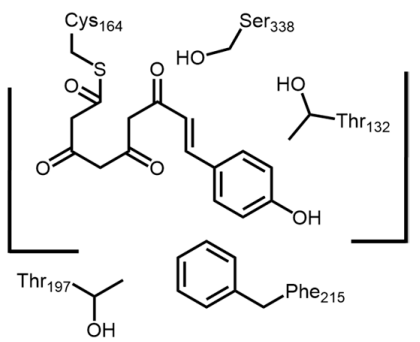

Figure 6. Comparison of the active sites of M. sativa CHS and R. palmatum BAS. (A) Synthesis of benzalacetone; (B) Schematic representations of the active site of BAS and CHS. The presence of Leu-208, Leu-125, and Cys-190 and the reorientation of Ser-331 in BAS changed the orientation of the starter acyl-CoA, $p$-coumaroyl-CoA, as well as the orientation of the growing polyketide intermediate, leading to the formation of benzalacetone.

\subsection{Other Members of Type III PKS from Plants}

\subsection{1. $p$-Coumaroyl Triacetic Acid Synthase and Stilbenecarboxylate Synthase}

p-Coumaroyl triacetic acid synthase (CTAS) from Hydrangea macrophylla var. thunbergii was found to catalyze three consecutive decarboxylative condensations of malonyl-CoA with a $p$-coumaroyl-CoA starter without cyclization, to form $p$-coumaroyl triacetic acid (14) (Scheme 3) [41]. A comparison 
between the amino acid sequences of $H$. macrophylla CHS and CTAS showed 76\% identity and only 12 amino acid residues which were significantly different in charge and size. Amongst them, replacing any of the nucleophilic residues in the CHS consensus with neutral amino acid (D/E18G) and amides (E264Q, D321N, and E324Q) in CTAS might have led to the loss of cyclization activity needed for the CHS reaction. Furthermore, the CHS-conserved Thr197, which is postulated to play a role in Claisen condensation, is replaced by an asparagine residue in CTAS [24]. All these residues are promising targets for site-directed mutagenesis and such studies may provide valuable insights into product specificity.

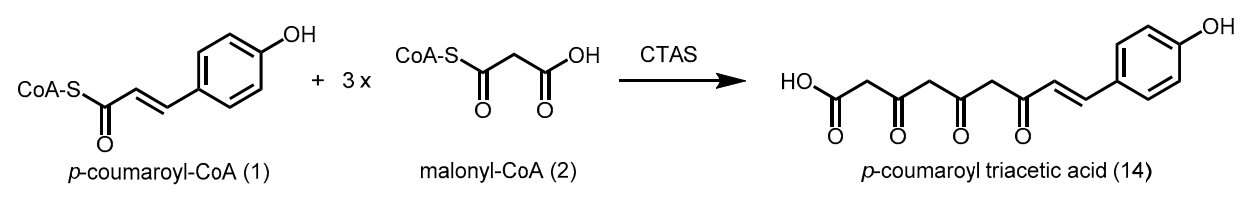

Scheme 3. Synthesis of $p$-coumaroyl triacetic acid catalyzed by $p$-coumaroyl triacetic acid synthase (CTAS) from Hydrangea macrophylla var. thunbergii.

Stilbenecarboxylate synthase (STCS) from another Hydrangea variety, H. macrophylla L., was found to utilize $p$-hydroxyphenylpropionyl-CoA as a starter and carry out three consecutive decarboxylative condensations of malonyl-CoA, followed by a C2 to $C 7$ aldol condensation with retention of the terminal carboxyl group to form 5-hydroxylunularic acid (15) (Scheme 4) [42]. Although not much is known about the mechanism of action, the identification of this new class of type III PKS adds possibilities to direct the synthesis of new polyketides.

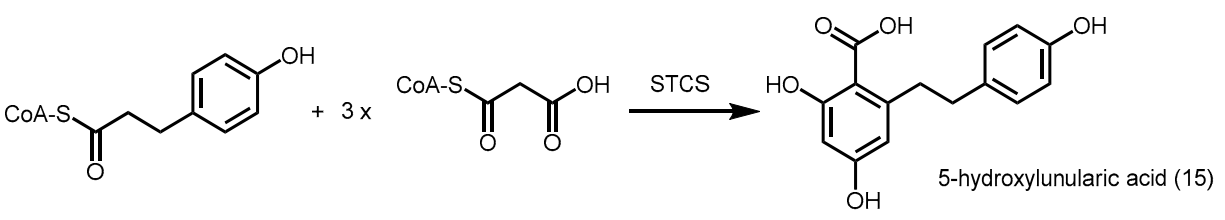

Scheme 4. Synthesis of 5-hydroxylunularic acid catalyzed by STCS from H. macrophylla L.

\subsubsection{Bibenzyl Synthase}

Bibenzyl synthase (BBS) cloned from a Phalaenopsis orchid utilizes $m$-hydroxyphenylpropionyl-CoA as a starter unit and catalyzes the decarboxylative condensation of three malonyl-CoA units, followed by an aldol condensation and decarboxylation to give 3,3',5-trihydroxybibenzyl (16) (Scheme 5) [43].

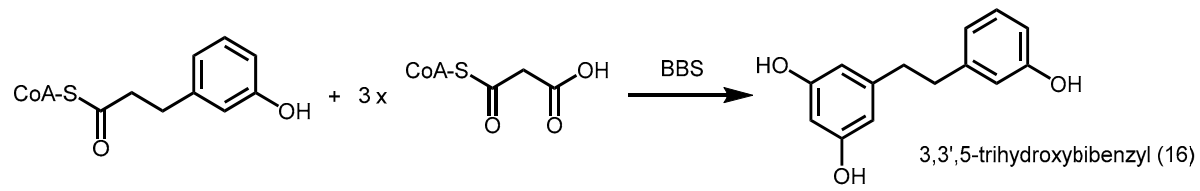

Scheme 5. Synthesis of 3,3',5-trihydroxybibenzyl catalyzed by BBS from a Phalaenopsis orchid.

A comparison of amino acid sequences revealed that BBS preferred $m$-substituted derivatives of dihydrocinnamoyl-CoA to their cinnamoyl-CoA counterparts because Val98 is replaced by alanine in BBS. This substitution is also seen in other divergent type III PKSs, such as Hordeum vulgare (barley) homoeriodictyol/eriodictyol synthase and Ruta graveolens acridone synthase, which are known to accept starters containing $m$-substituted phenyl rings [24]. Furthermore, Gly211 is replaced by threonine in BBS: this small-to-large mutation is thought to alter the shape of the coumaroyl-binding pocket, as this residue is situated at the front of the active site cavity, just beneath the 'gatekeeper' phenylalanine positioned at the end of the CoA-binding tunnel. Derivatives of dihydrocinnamoyl-CoA possess reduced propanoid moieties and have increased conformational flexibility, giving them more advantage over their rigid counterparts in binding to a contorted coumaroyl-binding pocket in BBS. 
The flexibility of the tetraketide intermediate due to saturation of the propanoid moiety may also aid it to attain a folded conformation suitable for cyclization more easily within a slightly contorted active site. More structural studies are needed to provide insights into the substrate selectivity and mode of cyclization of the intermediates in BBS.

\subsubsection{Benzalacetone Synthase}

Unlike M. sativa CHS, benzalacetone synthase (BAS) from R. palmatum is unable to generate the truncation products of the CHS reaction, bisnoryangonin and CTAL. Instead, BAS catalyzes the one-step decarboxylative condensation of malonyl-CoA to $p$-coumaroyl-CoA, followed by another decarboxylation to form benzalacetone ( $p$-hydroxyphenylbut-3-ene-2-one) (17) (Figure 6A) [44]. The biosynthesis of benzalacetone in R. palmatum was attributed to the replacement of the 'gatekeeper' Phe215 with Leu208 in BAS. In alfalfa CHS, Phe215 is hypothesized to aid in orientingsubstrates during polyketide chain extension. Thus, a replacement with leucine in BAS may prevent subsequent chain extension of the diketide intermediate. In addition, the rhubarb BAS (PDB ID: 3A5R) uses an alternative coumaroyl-binding pocket to orient the starter-CoA, as the entrance to the original coumaroyl-binding pocket of the alfalfa CHS is blocked sterically in BAS by Leu125, Leu208 and Ser331 (Figure 6B) [45,46]. A single large-to-small mutation from leucine to threonine at residue position 125 of BAS back to the CHS conserved residue was found to be sufficient to unlock the buried coumaroyl-binding pocket, and the L125T BAS mutant was able to generate naringenin, CTAL and bisnoryangonin, in addition to benzalacetone [46]. Unlike STS, the crystal structure of rhubarb BAS also pointed to a hydrogen bond network between the Cys-His-Asn catalytic triad and a putative nucleophilic water molecule thought to play a role in thioester bond cleavage and the final decarboxylation step [45]. These findings provided a novel structural basis for the cleavage of the thioester bond of the enzyme-bound intermediate and the subsequent decarboxylation to form benzalacetone.

The crystal structure of PcPKS1 from Polygonum cuspidatum (Japanese knotweed) was also recently solved, and studies showed PcPKS1 to have both CHS and BAS activities [47]. Unlike rhubarb BAS and the classical BAS PcPKS2, the 'gatekeeper' Phe215 remained in PcPKS1, thus suggesting a different catalytic mechanism in the bifunctional PCPKS1. More engineering studies are needed to shed light on the functional divergence of this unique PKS.

\subsubsection{Type III Polyketide Synthases from Curcuma longa}

The biosynthesis of curcuminoids in C. longa (turmeric) has been proposed to involve two type III PKSs. Diketide-CoA synthase (DCS) catalyzes the decarboxylative condensation of malonyl-CoA with feruloyl-CoA (18) to give rise to feruloyldiketide-CoA (19) (Scheme 6A). DCS is unlike most type III PKSs as it is able to release the CoA product without cleaving the thioester linkage. Subsequently, curcumin synthase (CURS) catalyzes the decarboxylative condensation of feruloyldiketide acid (20), the hydrolyzed $\beta$-keto acid of feruloyldiketide-CoA, with another unit of feruloyl-CoA starter to produce curcumin (21) (Scheme 6B) via a rarely observed head-to-head condensation of ketide units. In addition, CURS is also able to catalyze the formation of curcumin from feruloyl-CoA and malonyl-CoA, albeit at a much slower rate [48].

(A)

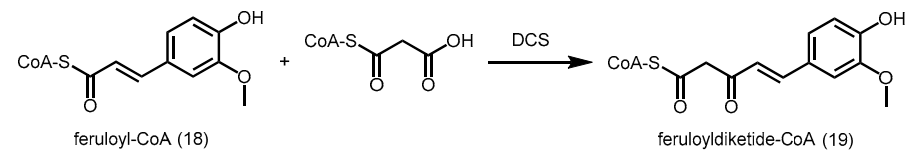

(B)

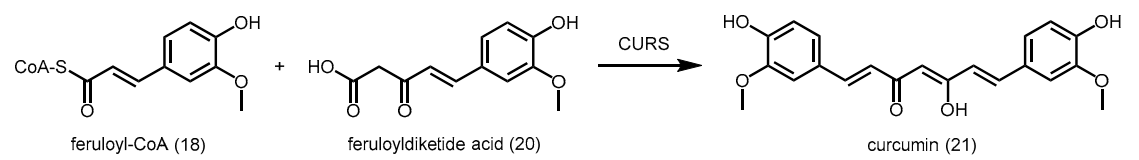

Scheme 6. Bioynthesis of curcuminoids in C. longa. (A) DCS catalyzes the formation of feruloyldiketide$\mathrm{CoA}$, one of the rare ketides with a CoA still attached to the final product; (B) CURS catalyzes the formation of curcumin from feruloyl-CoA and feruloyldiketide via a rare head-to-head condensation. 
Interestingly, curcuminoid synthase (CUS) from Oryza sativa is able to generate bisdemethoxycurcumin (22) from the decarboxylative condensation of two units of $p$-coumaroyl-CoA and one unit of malonyl-CoA, via a $p$-coumaroyldiketide acid intermediate (Figure 7A). Although the Cys-His-Asn catalytic triad and most conserved residues are present in the CUS active site, the crystal structure of CUS (PDB ID: 3ALE) revealed a downward expanding active site that is large enough to accommodate two starter CoAs and one extender CoA (Figure 7B) [49].

(A)

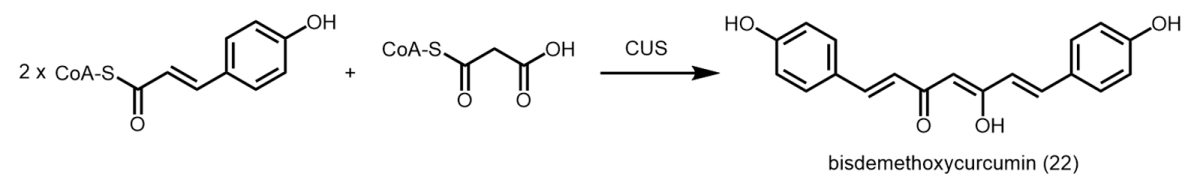

(B) CUS

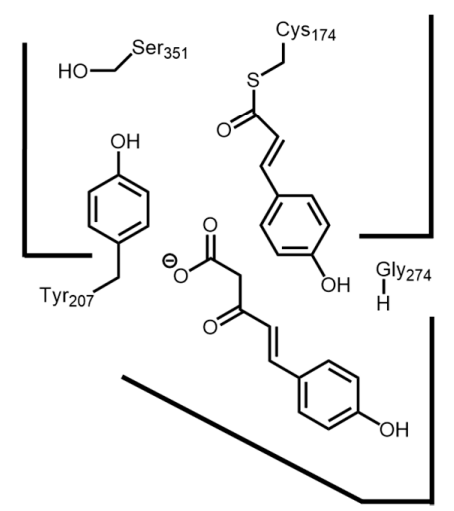

CHS

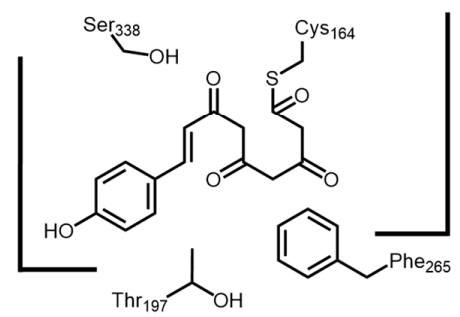

Figure 7. Comparison of the active sites of M. sativa CHS and O. sativa CUS. (A) CUS catalyzes the condensation between two units of $p$-coumaroyl-CoA and one unit of malonyl-CoA to form bisdemethoxycurcumin; (B) Schematic representations of the active sites of CUS and CHS. The presence of glycine at position 274 and tyrosine at position 207 orients the active site to allow longer polyketides to be generated.

Further analysis highlighted that the CHS-conserved residues, Thr132, Thr197, Gly256, and Phe265 were replaced by Asn142, Tyr207, Met265, and Gly274, respectively in CUS. This offers no surprise as Thr132 plays a role in aldol condensation [26,31], while Thr197 is thought to direct Claisen condensation [24], and both Thr197 and Gly256 are able to direct polyketide chain lengths in other type III PKSs [35-40]. Hence by replacing these residues, the product specificity of CUS is likely to be very different from other type III PKSs. Conformational differences due to these amino acid changes resulted in a loss of the coumaroyl-binding pocket in CUS. As for Phe265, it is located near the CoA-binding tunnel in alfalfa CHS, therefore a less bulky F265G mutation in CUS is able to allow larger substrates to enter the active site [49]. In addition, the smaller glycine residue opens a gate to a downward expanding pocket, which is thought to accommodate the $p$-coumaroyldiketide acid intermediate before the second decarboxylative condensation with another unit of $p$-coumaroyl-CoA.

The crystal structure of CUS revealed a putative nucleophilic water molecule which could form a hydrogen bond network consisting of Ser351-Asn142- $\mathrm{H}_{2} \mathrm{O}-\mathrm{Tyr} 207-\mathrm{Glu} 202$ near the catalytic Cys174 residue in the active site (Figure 8). This is similar to the Glu192-Thr132- $\mathrm{H}_{2} \mathrm{O}-\mathrm{Ser} 338$ "aldol-switch" hydrogen bond network in STS, which participates in the cleavage of the thioester bond of the cysteine-bound linear tetraketide intermediate. The hydrogen bond network in CUS is postulated to be important for the termination of the polyketide chain extension at the diketide stage by cleaving the thioester bond of the cysteine-bound intermediate so as to produce $p$-coumaroyldiketide acid, which serves as the second extender substrate [49]. These findings provide valuable insights into the mechanism of action of CUS, the first type III PKS capable of utilizing two starters and one extender $\mathrm{CoA}$ to generate polyketides. 


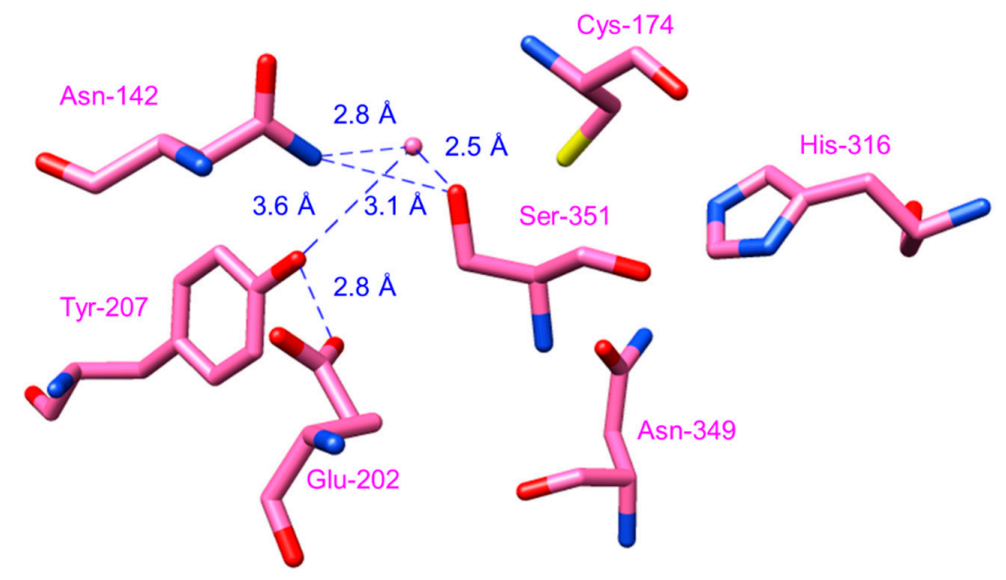

Figure 8. Hydrogen-bond network of CUS (PDB ID: 3ALE) consisting of Ser351, Asn142, Tyr207, Glu202, and a water molecule near the catalytic Cys174.

\subsubsection{Benzophenone Synthase and Biphenyl Synthase}

Among the starter-CoAs utilized by plant PKSs, benzoyl-CoA (23) is a rare starter unit greatly preferred only by two kinds of type III PKSs, benzophenone synthase (BPS) and biphenyl synthase (BIS) [50]. In cell cultures of Hypericum androsaemum (St. John's wort family of plants), BPS catalyzes the decarboxylative condensation of three units of malonyl-CoA with one unit of benzoyl-CoA to form a tetraketide intermediate, which undergoes an intramolecular $\mathrm{C} 6$ to $\mathrm{C} 1 \mathrm{Claisen}$ condensation to give 2,4,6-trihydroxybenzophenone (25, Scheme 7) [51]. Intriguingly, H. androsaemum CHS showed $60 \%$ identity to BPS, and was able to utilize benzoyl-CoA as a starter with low activity to form benzophenone. However, BPS was unable to have any detectable activity with derivatives of cinnamoyl-CoA. Mutagenesis of three CHS residues lining the active site to their corresponding counterparts in BPS (L263M/F265Y/S338G) resulted in a mutant $H$. androsaemum CHS which preferred benzoyl-CoA to $p$-coumaroyl-CoA. This suggests a combination of amino acid changes can affect the coumaroyl-binding pocket of $\mathrm{CHS}$, resulting in a greater substrate preference for benzoyl-CoA. Identical replacements (L263M/F265Y/S338G) were also found in the functionally similar Garcinia mangostana L. (mangosteen) BPS [52]. In cell cultures of the medicinal herb Centaurium erythraea, 3-hydroxybenzoyl-CoA (24) is the preferred starter CoA for BPS, giving rise to 2,3',4,6-tetrahydroxybenzophenone (26, Scheme 7), the precursor of xanthones [53]. More structural studies are needed to provide insights as to why BPS is unable to utilize phenylpropanoid-CoA esters as starters.<smiles>[X]c1ccc(C(=O)O[Na])cc1</smiles>

$\mathrm{X}=\mathrm{H}$, benzoyl-CoA (23) $\mathrm{X}=\mathrm{OH}$, 3-hydroxybenzoyl-CoA (24)

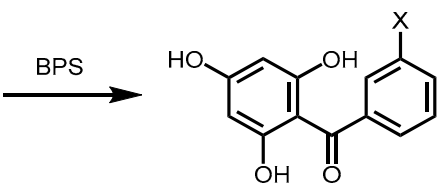

$\mathrm{X}=\mathrm{H}, 2,4,6$-trihydroxybenzophenone (25) $\mathrm{X}=\mathrm{OH}, 2,3$, 4,6-tetrahydroxybenzophenone (26)

Scheme 7. Benzophenone synthesis catalyzed by BPS from H. androsaemum $(\mathrm{X}=\mathrm{H})$ and C. erythraea $(\mathrm{X}=\mathrm{OH})$.

Similar to BPS, BIS from yeast extract-treated cell cultures of Sorbus aucuparia forms a tetraketide intermediate from one unit of benzoyl-CoA and three units of malonyl-CoA. However, the intermediate undergoes a C2 to $\mathrm{C} 7$ aldol condensation instead of a Claisen condensation, followed by a decarboxylation to give 3,5-dihydroxybiphenyl (27, Scheme 8) [54]. 


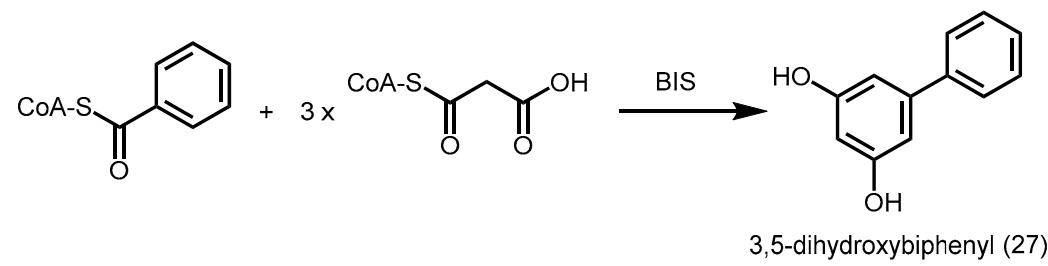

Scheme 8. Biosynthesis of 3,5-dihydroxybiphenyl catalyzed by S. aucuparia BIS.

BIS was also not reactive towards derivatives of cinnamoyl-CoA, while S. aucuparia CHS was able to accept benzoyl-CoA to give the derailment product, benzoyldiacetic acid lactone. Although both STS and BIS carry out aldol condensation, Thr132 and Ser338 are replaced by alanine and glycine respectively in BIS. Hence, the "aldol-switch" hydrogen bond network consisting of Glu192-Thr132- $\mathrm{H}_{2} \mathrm{O}-\mathrm{Ser} 338$ is absent in BIS. Furthermore, subtle point mutations used to disrupt this hydrogen bond network in a mutant CHS with STS activity (18xCHS), revealed that a T132A mutation led to the production of more chalcones instead of stilbenes [26]; this was surprisingly not observed in BIS and suggested a different mode of catalysis in BIS.

\subsubsection{Acridone Synthase and Quinolone Synthase}

In addition to the C-C bond forming reactions, two groups of type III PKSs catalyze the C-N bond formation, resulting in the production of anthranilate-derived alkaloids. Acridone synthase (ACS) from cell cultures of $R$. graveolens catalyze the decarboxylative condensation of three units of malonyl-CoA with one unit of $\mathrm{N}$-methylanthraniloyl-CoA (28), followed by a Claisen condensation to give $\mathrm{N}$-methylaminobenzophenone, and a second ring closure to give 1,3-dihydroxy- $\mathrm{N}$-methylacridone (30, Scheme 9) [55]. Although $R$. graveolens ACS share $74 \%$ amino acid sequence identity with $R$. graveolens CHS, ACS was only able to utilize $p$-coumaroyl-CoA with low activity $(10 \%-20 \%)$ to form naringenin. On the other hand, $R$. graveolens CHS was unable to accept the bulky $N$-methylanthraniloyl-CoA as a starter [56].

Quinolone synthase (QNS) from Aegle marmelos was able to catalyze the decarboxylative condensation of one unit of malonyl-CoA with one unit of $\mathrm{N}$-methylanthraniloyl-CoA to form a diketide, which spontaneously cyclizes by amide formation to give 4-hydroxy- $N$-methylquinolone (29, Scheme 9). QNS was also able to form benzalacetone from $p$-coumaroyl-CoA and malonyl-CoA. Homology modeling revealed that the CoA-binding tunnel, the Cys-His-Asn catalytic triad, and most of the active site residues are maintained in A. marmelos QNS. However, the CHS-conserved residues Thr132, Ser133, and Phe265 are replaced by Ser132, Ala133, and Val265, respectively [57]. These substitutions are also present in $R$. graveolens ACS, hence, they are likely to play an important role in starter unit specificity [58]. The decrease in steric bulk of the residues at these three positions results in a larger active site cavity that can accommodate the bulkier $N$-methylanthraniloyl-CoA. Site-directed mutagenesis studies showed that a triple ACS mutant (S132T/A133S/V265F) was transformed into a functional CHS with some ACS side activity. Intriguingly, a similar triple QNS mutant (S132T / A133S/V265F) had no activity, but a double QNS mutant (S132T/A133S) was converted into a functional CHS that utilized $p$-coumaroyl-CoA instead of $N$-methylanthraniloyl-CoA, and the number of elongation steps was increased, leading to the production of naringenin [57]. This suggests slight differences in the active site architectures of ACS and QNS.

Recently, the crystal structures of ACS (PDB ID: 3WD7) and QNS (PDB ID: 3WD8) from Citrus microcarpa were solved and wide active site entrances were found in both PKSs, which enabled the access of the bulky N-methylanthraniloyl-CoA [59]. The V98A substitution, hypothesized to be important for $m$-substituted phenyl substrate selectivity [24], was only found in R. graveolens ACS [58] but not in the other ACS and QNSs identified so far [59]. C. microcarpa ACS was found to be a highly promiscuous enzyme with a large active site cavity, being able to accept different starter CoAs to generate chalcone, benzophenone, and phloroglucinol scaffolds in addition to the production of 4-hydroxy- $N$-methylquinolone (29), 1,3-dihydroxy- $N$-methylacridone (30), and $\mathrm{N}$-methyl-anthraniloyltriacetic acid lactone (31, Scheme 9). 


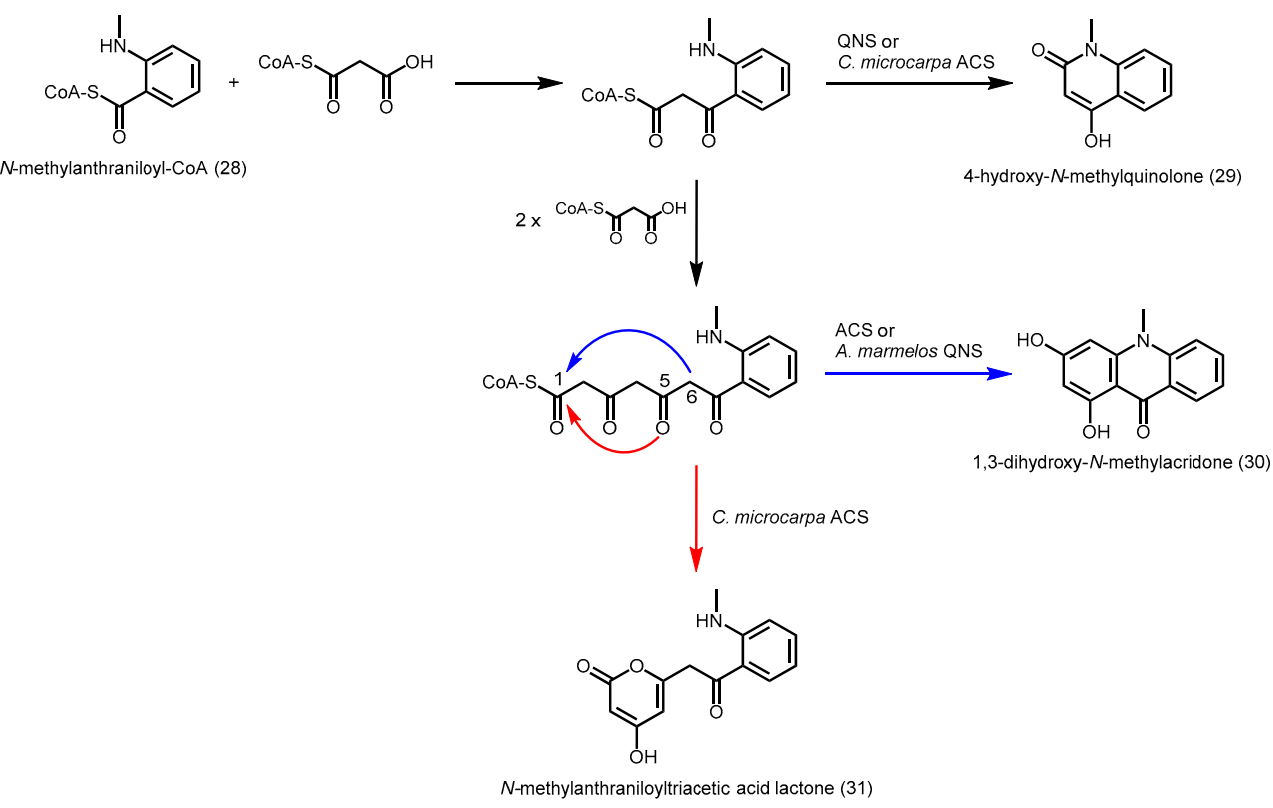

Scheme 9. Synthesis of anthranilate-derived alkaloids catalyzed by R. graveolens ACS (30 only), A. marmelos QNS (29 and 30), C. microcarpa QNS (29 only), and C. microcarpa ACS (29, 30, and 31).

C. microcarpa QNS can only produce the diketide quinolone as the sole product, unlike A. marmelos QNS. Compared to R. graveolens ACS and A. marmelos QNS, similar replacements were found in C. microcarpa ACS (T132S/S133A/F265V), but not in C. microcarpa QNS (T132M/S133A/F265L). The volume of the active site cavity of C. microcarpa QNS (Figure 9A) is smaller compared to R. palmatum BAS (Figure 9B), due to the presence of methionine residues at positions 132 and 194, and tyrosine at position 197. These residues block the access to the coumaroyl-binding pocket [59]. Hence, C. microcarpa QNS is unable to utilize p-coumaroyl-CoA as a substrate, and chain elongation with $\mathrm{N}$-methylanthraniloyl-CoA cannot proceed beyond the diketide stage. Intriguingly, a large-to-small tyrosine to alanine mutation at residue position 197 in C. microcarpa QNS mutant was able to gain the production of benzalacetone and bisnoryangonin. Site directed mutagenesis of the important active site residues Ser132, Thr194, and Thr197 in C. microcarpa ACS to Met, Met, and Tyr respectively resulted in a quinolone-producing enzyme which no longer produces acridone, $\mathrm{N}$-methylanthraniloyltriacetic acid lactone, and chalcone. These results provided the first structural basis for the generation of anthranilate-derived alkaloids by type III PKSs.
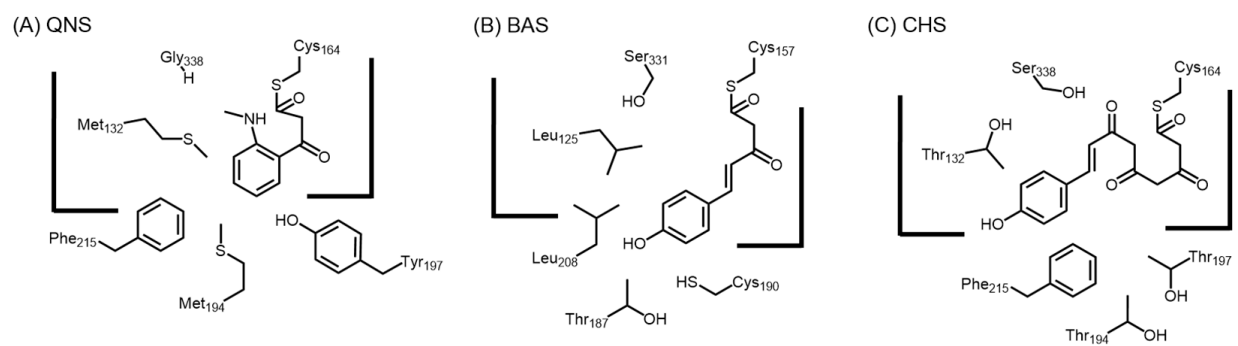

Figure 9. Comparison of the active sites (A) C. microcarpa QNS; (B) R. palmatum BAS; and (C) M. sativa CHS. The presence of Tyr197 and Met194 in QNS greatly reduces the active site compared to BAS and CHS; hence, only a diketide may be formed.

\subsubsection{Phloroglucinol Synthase, Alkylresorcinol Synthase, and Alkylpyrone Synthase}

Most plant type III PKSs have evolved to use CoA-esters derived from phenylpropanoid metabolism as starters, but some functionally divergent PKSs are adapted to utilizing long-chain 
acyl-CoAs. Previous studies on Petroselinum hortense (parsley) CHS in the 1980s showed that type III PKSs can be promiscuous enzymes [22]. In addition to the natural substrate $p$-coumaroyl-CoA, parsley CHS was able to utilize several aliphatic CoA esters as starters, such as butyryl-CoA and hexanoyl-CoA, which were converted efficiently to acylphloroglucinols. Nevertheless, these short chain fatty acyl-CoA esters are unlikely to occur at the site of flavonoid biosynthesis, and the purpose of having activity with these substrates remained unknown. Recently, phloroglucinol synthase (PhID) from Ectocarpus siliculosus (brown alga) was found to be able to synthesize phloroglucinol (32) from three units of malonyl-CoA (Scheme 10A), and a positive correlation was identified between reacclimation to seawater and the phloroglucinol content in cells [60]. The phloroglucinol synthase was also able to catalyze the decarboxylative condensation of three units of malonyl-CoA with one unit of lauroyl-CoA $(n=9)$ or palmitoyl-CoA $(n=13)$ to form tetraketide $\alpha$-pyrones 33 and acylphloroglucinols 34 (Scheme 10B).

(A)

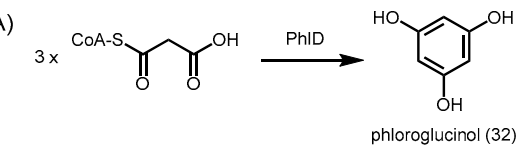

(B)
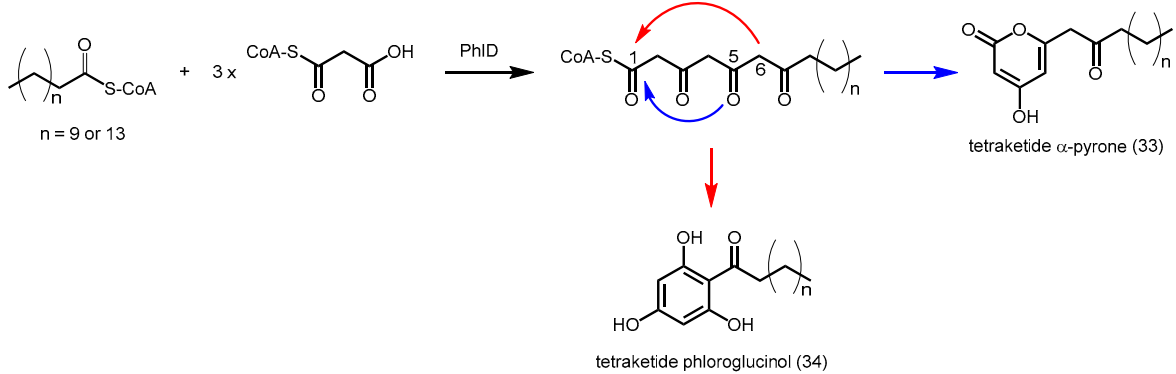

Scheme 10. Synthesis of phloroglucinols and $\alpha$-pyrones catalyzed by PhlD from E. siliculosus. (A) PhlD utilizes three units of malonyl-CoA to form the simplest phloroglucinol; (B) PhID also accepts longer acyl-CoAs such as lauryl-CoA $(n=9)$ or palmitoyl-CoA $(n=13)$ to form their respective tetraketide $\alpha$-pyrone and tetraketide phloroglucinol.

PhlD was co-crystallized with arachidonic acid and the structure of the enzyme was solved at $2.85 \AA$ resolution using molecular replacement (PDB ID: 4B0N). The structure showed that an acyl-binding tunnel is present instead of a coumaroyl-binding pocket, hence enabling the PKS to accommodate long-chain acyl-CoAs (Figure 10) [60]. The residues in the acyl-binding tunnel are not conserved in M. sativa CHS; when arachidonic acid is superimposed on CHS, clashes between the residues lining the site and substrate were observed (Figure 10, indicated by purple bonds of the residues).
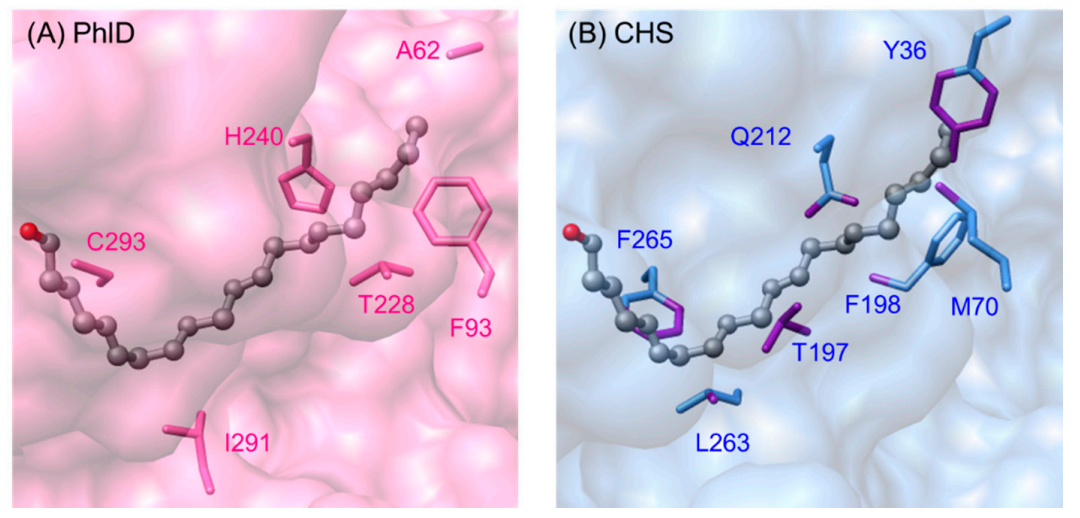

Figure 10. Surface view of arachidonic acid-(A) on the acyl-binding tunnel in E. siliculosus PhID (PDB ID: 4B0N) and (B) superimposed on M. sativa CHS (PDB ID: 1CHW). Clashes between the corresponding residues in CHS that line the "tunnel" and arachidonic acid were observed (indicated by purple bonds on the residues). 
An extra pocket is also present at the dimeric interface of the enzyme, when compared to the structures of CHS and STS. This is due to the replacement of a bulky aromatic amino acid in the cavity with Gly190 in brown alga phloroglucinol synthase, thus increasing the volume of the active site cavity. A higher resolution crystal structure is needed in order to shed more light on the molecular basis of this functionally divergent PKS.

Alkyl-resorcylic acid synthase 2 (ARAS2) from O. sativa catalyzes the synthesis of bis-5-alkylresorcinols using alkanedioic acid $N$-acetylcysteamine (NAC) dithioester (35) and malonyl-CoA (Scheme 11) [61]. One of the two NAC groups is first converted into a resorcinol group, generating an intermediate. ARAS2 subsequently catalyzes the condensation of malonyl-CoA with the intermediate and convert the remaining NAC group into another resorcinol group, yielding bis-5-alkylresorcinol (36, Scheme 11). This is analogous to the two-step reaction by curcuminoid synthase (CUS), although the mechanism of synthesis is different. ARAS2 uses two different starters and the same extenders for bis-5-alkylresorcinol biosynthesis, while CUS utilizes the same starters and two different extenders for the biosynthesis of bisdemethoxycurcumin. More structural studies are needed to provide insights into the unusual substrate specificities of ARAS2.

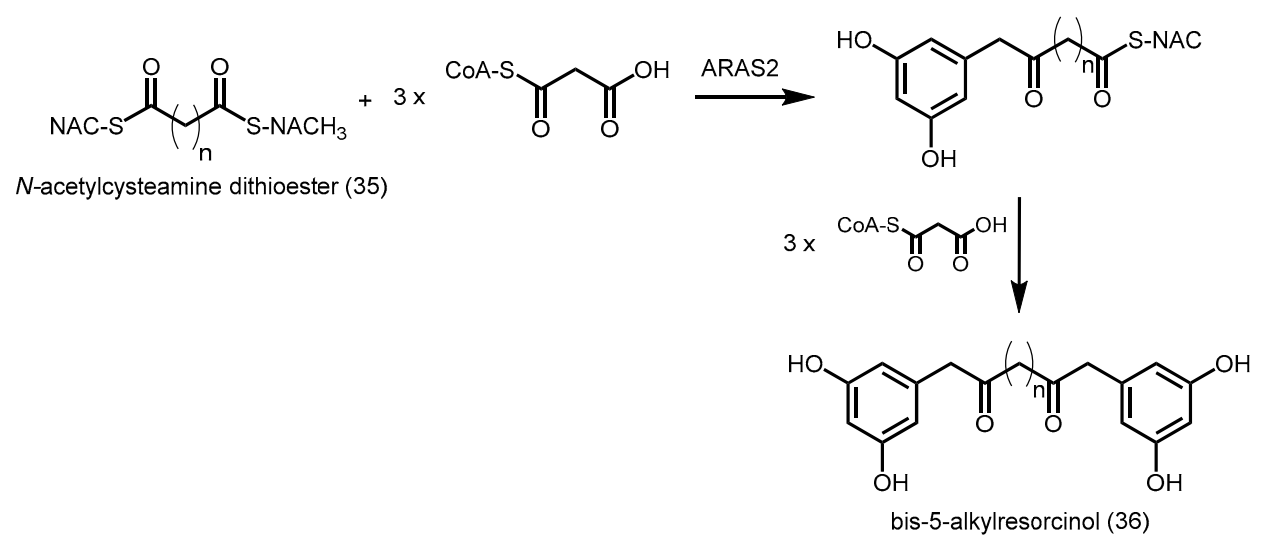

Scheme 11. Synthesis of bis-5-alkylresorcinol catalyzed by O. sativa ARAS2.

Recently, alkylpyrone synthases from Arabidopsis thaliana (PKSA and PKSB) were found to be able to produce triketide and tetraketide $\alpha$-pyrones 37 and 38 from the decarboxylative condensation of malonyl-CoA with medium-chain to long-chain, and hydroxylated fatty acyl-CoAs (Scheme 12) [62]. These tetraketide $\alpha$-pyrones are important for sporopollenin biosynthesis during pollen grain development, while the triketide $\alpha$-pyrones are thought to be derailment products. Although these PKSs are related to CHS, they do not display CHS activity. A similar anther-specific chalcone synthase-like enzyme from Physcomitrella patens (PpASCL) was also discovered; the enzyme catalyzes the condensation malonyl-CoA with saturated acyl-CoAs (C6-C20), unsaturated acyl-CoAs (C16:1 or C18:1) or hydroxyl fatty acyl-CoAs to generate alkyl and hydroxyalkyl $\alpha$-pyrones that are important for moss spore wall formation [63].

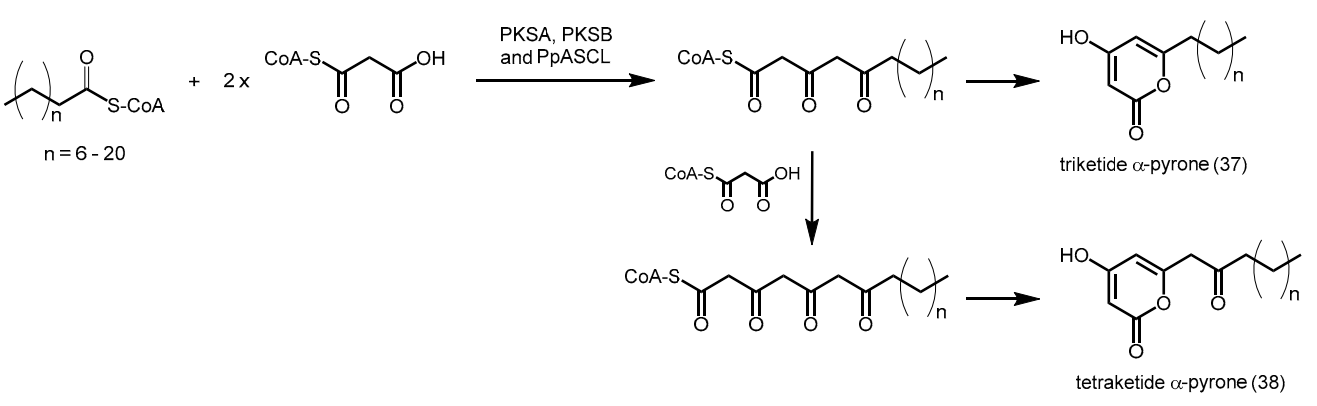

Scheme 12. Synthesis of triketide and tetraketide $\alpha$-pyrones catalyzed by $A$. thaliana PKSA and PKSB and P. patens PpASCL. 
Both PKSA and PpASCL are also able to react with $p$-coumaroyl-CoA to yield bisnoryangonin. Based on ab initio multiple-threading and molecular dynamics simulation methods [63], a bulkier residue like Thr197 in alfalfa CHS is replaced by a smaller Gly205 in PKSA and Gly225 in PpASCL, hence potentially widening the entrance of the acyl-binding tunnel. In addition, the conserved Gln212 in alfalfa CHS is substituted by Ala220 in PKSA and Ala240 in PpASCL. The inability of alanine to form multiple hydrogen bonds with other active site residues may provide the structural flexibility for a larger active site cavity, thus enabling these PKSs to play a role in lipid metabolism.

\subsection{Bacterial Type III PKSs}

\subsubsection{1,3,6,8-Tetrahydroxynaphthalene Synthase (THNS)}

Unlike plant type III PKSs, bacterial type III PKSs usually share less than 50\% identity with each other and with plant type III PKSs. Bacterial type III PKSs were first characterized from the Gram-positive Streptomyces griseus. RppA showed 30\% identity to plant CHSs, and was able to utilize malonyl-CoA as a starter and catalyze the decarboxylative condensation of another four units of malonyl-CoA to form a pentaketide, which undergoes dual cyclization reactions and decarboxylation to generate 1,3,6,8-tetrahydroxynaphthalene (THN) (39) [16]. THN can be further oxidized non-enzymatically to yield a reddish compound, flaviolin (40, Scheme 13). Cys138 serves as the catalytic residue of RppA, since a C138S RppA mutant was found to have no enzyme activity.

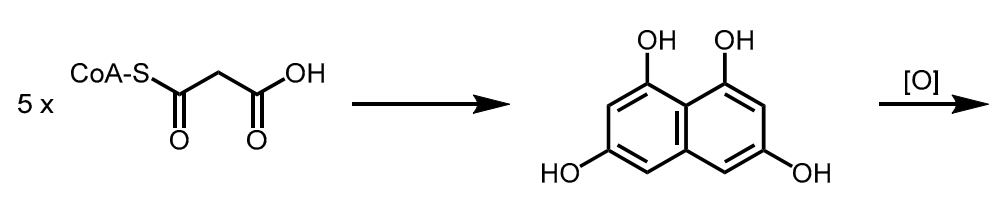

1,3,6,8-tetrahydroxynaphthalene (39)

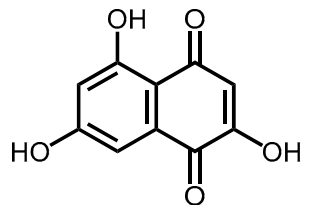

flaviolin (40)

Scheme 13. Synthesis of 1,3,6,8-tetrahydroxynaphthalene and flaviolin by S. griseus RppA.

RppA also has alkylpyrone synthase and phloroglucinol synthase activities. It accepts aliphatic acyl-CoAs (C4-C8) as starters and catalyze decarboxylative condensations of malonyl-CoA to give $\alpha$-pyrones and phloroglucinols [64]. The active site cavity of RppA is bigger than most type III PKSs; it can catalyze the formation of a hexaketide, 4-hydroxy-6-(2', 4',6 $6^{\prime}$-trioxotridecyl)-2-pyrone, using one unit of octanoyl-CoA and five units of malonyl-CoA. However unlike most plant PKSs, RppA is unable to accept aromatic acyl-CoAs such as benzoyl-CoA and phenylacetyl-CoA, indicating differences in the active site cavities of RppA and plant PKSs.

A similar THNS was also found in Streptomyces coelicolor A3(2), although the malonyl-CoA binding for THNS was less efficient compared to that of the S. griseus RppA [65]. The crystal structure of S. coelicolor A3(2) THNS (PDB ID: 1U0M) reveals a cryptic tunnel that extends into the 'floor' of the traditional type III PKS active site cavity [66]. This tunnel enables the selective expansion of available active site volume by allowing the aliphatic tail of starter-derived polyketide intermediates to be sequestered. In addition, the distance between Cys138 and the cryptic tunnel precludes all but the longest reaction intermediates from accessing the novel tunnel. This provides a feasible structural explanation for the ability of RppA and THNS to catalyze an extra polyketide elongation step, instead of fewer polyketide elongation steps, when primed with a longer starter such as octanoyl-CoA in vitro. In addition to the cryptic tunnel, less bulky residues compared to alfalfa CHS were identified at several positions in the active site, including T132C, T194C, T197C, and S338A [66]. However, Gly256 in alfalfa CHS is replaced by Tyr224 in THNS, resembling the 'horizontally restricting' G256L substitution seen in 2-pyrone synthase (2-PS), pentaketide chromone synthase (PCS), aloesone synthase (ALS), and octaketide synthase (OKS), which utilize acetyl-CoA instead of $p$-coumaroyl-CoA as starters. The 'downward expanding' conformation of THNS active site due to the cryptic tunnel and T197C substitution, and the 'horizontally restricting' G256Y substitution provide some insights into the ability of THNS to utilize a small starter, and yet have the required volume for the extra polyketide 
elongation steps and dual cyclization reactions needed for THN generation (Figure 11). Furthermore, the replacement of Thr132 in alfalfa CHS with Cys106 in THNS leads to an additional cysteine residue in the active site, which may facilitate polyketide elongation beyond the triketide stage. We will need more liganded enzyme structures with substrate, polyketide intermediates, or product before more information regarding the substrate specificity, polyketide chain length control, and mode of dual cyclization of THNS can be ascertained.

(A) THNS

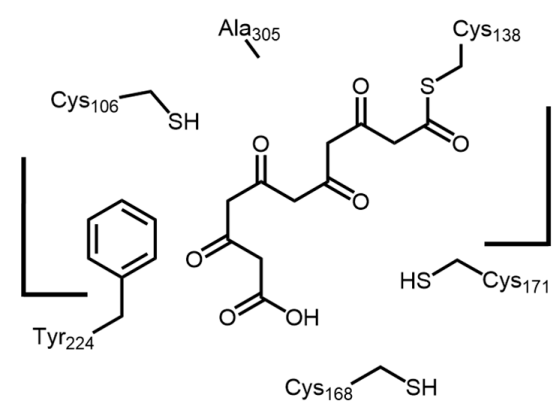

(B) $\mathrm{CHS}$

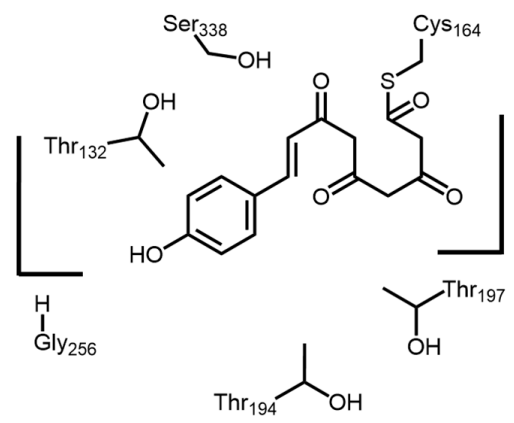

Figure 11. Schematic representation of the active sites of (A) S. coelicolor THNS and (B) M. sativa CHS.

\subsubsection{Phloroglucinol Synthase from Pseudomonas fluorescens}

Phloroglucinol synthase from Pseudomonas fluorescens (PfPhlD) catalyzes the synthesis of phloroglucinol, a precursor of the anti-fungal 2,4-diacetylphloroglucinol (Scheme 10A). It has the same activity as E. siliculosus PhlD and about 40\% identity with RppA [17,67]. PfPhlD was also found to display broad substrate specificity and formed $\alpha$-pyrones when incubated with starters such as C4-C12 aliphatic acyl-CoAs and phenylacetyl-CoA. Similar to RppA, when primed with medium to long-chain acyl-CoAs like hexanoyl-CoA, octanoyl-CoA, and decanoyl-CoA, PhlD catalyzed extra polyketide extension steps to generate up to heptaketide products.

Homology modeling of PfPhID using the structure of THNS as a reference highlighted the presence of a similar buried tunnel extending into the 'floor' of the active site cavity, which can potentially aid the binding of long chain acyl-CoAs [68]. The different reactivity towards phenylacetyl-CoA might suggest a larger and more flexible tunnel in PfPhlD that is capable of incorporating the bulky starter, compared to RppA. Saturation mutagenesis was done on important residues lining the buried tunnel, by replacing the codon encoding the residue to be mutated with the sequence 'NNS'. This substitution with 'NNS' allows the replacement of the residue with all 20 amino acids, while reducing the number of premature stop codons to one (TAG) out of three. After deconvolution, three mutations M21I, H24V, and L59M, were found to significantly decrease the reactivity of PfPhID with lauroyl-CoA, but not its physiological activity to synthesize phloroglucinol. This showed that even subtle decreases in the tunnel volume could impair the ability of PhlD to accept long chain acyl-CoAs. This highlighted the utility of a combination of rational design and random mutagenesis in changing substrate specificities, suggesting novel strategies for the combinatorial biosynthesis of unnatural polyketide libraries.

\subsubsection{3,5-Dihydroxyphenylacetic Acid Synthase}

3,5-Dihydroxyphenylacetic acid synthase from Amycolatopsis mediterranei (DpgA) catalyzes the decarboxylative condensation of four units of malonyl-CoA, followed by a likely C8 to C3 aldol condensation to yield 3,5-dihydroxyphenylacetic acid (41, Scheme 14). The compound is a precursor of (S)-3,5-dihydroxyphenylglycine, an amino acid utilized in the biosynthesis of balhimycin, a vancomycin derivative [18].

Intriguingly, site-directed mutagenesis of the conserved catalytic Cys160 to alanine or serine in DpgA did not completely ablate the ability to form products [69]. When malonyl-diketidyl-CoA was 
used to probe how polyketide elongation and cyclization of the polyketide intermediate occurs, DpgA was found to accept malonyl-diketidyl-CoA, both as a starter and an extender, thus complicating analyses [70]. More structural studies are needed to understand how polyketide extension occurs without the catalytic cysteine, and how the $C 8$ to $C 3$ aldol condensation, which is unique to DpgA, is catalyzed.

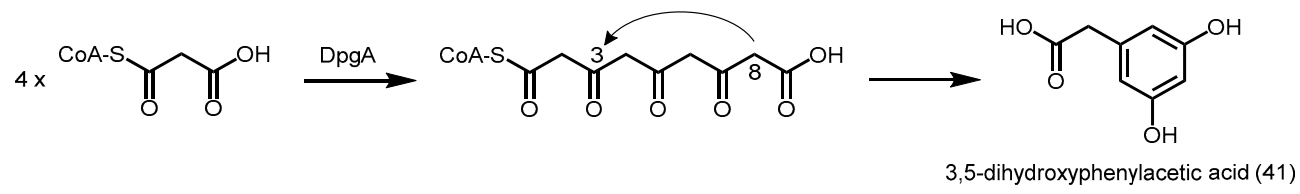

Scheme 14. Synthesis of 3,5-dihydroxyphenylacetic acid catalyzed by A. mediterranei DpgA.

\subsubsection{Biosynthesis of Alkylpyrones by PKS11 and PKS18}

Similar to A. thaliana PKSA and P. patens PpASCL (anther-specific chalcone synthase-like enzyme), PKS11 and PKS18 from Mycobacterium tuberculosis were found to be able to accept medium to long-chain acyl-CoAs (C6-C20) to produce tetraketide and/or triketide $\alpha$-pyrones (Scheme 12) [71]. The crystal structure of PKS18 (PDB ID: 1TED)was independently determined at the same time as the THNS structure, and the orientations of the catalytic triad (Cys175, His313, Asn346) and other functional residues were found to be similar to that of $M$. sativa $C H S$. Subtle differences between the primary sequences of PKS18 and M. sativa CHS result in the protrusion of Cys205 in PKS18 (corresponding to Thr194 in alfalfa CHS) into the putative coumaroyl-binding pocket [72]. In contrast, PKS18 was found to have a novel acyl-binding tunnel, which can bind aliphatic acyl-CoAs as starter units (Figure 12). Comparison with alfalfa CHS crystal structure showed that the entrance of the binding tunnel in CHS was blocked by Thr197, whereas the corresponding Asn208 residue in PKS18 was not extending into the tunnel. This is analogous to PKSA and PpASCL, whereby a replacement of bulky Thr197 in alfalfa CHS with a smaller Gly205 in PKSA and Gly225 in PpASCL is believed to widen the entrance of the acyl-binding tunnel, enabling them to utilize larger acyl-CoAs as starters [63].
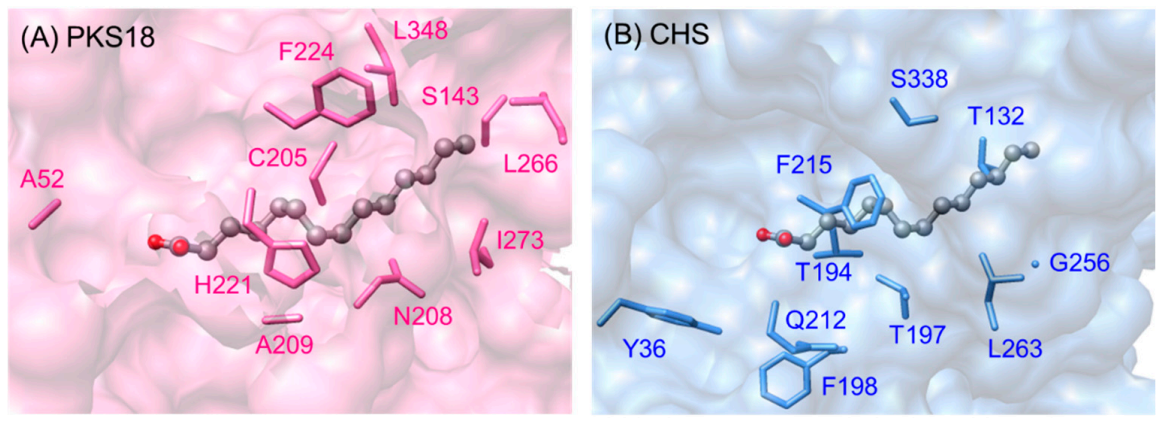

Figure 12. (A) Acyl-binding tunnel in M. tuberculosis PKS18 (PDB ID: 1TED); (B) Myristic acid (from PDB ID: 1TED) superimposed on $M$. sativa CHS (PDB ID: 1CGK). The residues on the acyl binding tunnel of PKS18 are relatively smaller (A52, S143, C205, A209) and/or more hydrophobic (N208, H221, L266, L348).

The crystal structure of PKS11 was recently solved (PDB ID: 4JAP), and a buried hydrophobic tunnel was also revealed, together with a CoA-binding tunnel [73]. Co-crystallizations and in vitro enzyme assays revealed that PKS11 can catalyze the decarboxylative condensation of methylmalonyl-CoA with palmitoyl-CoA (C16) or stearoyl-CoA (C18), followed by another decarboxylative condensation of malonyl-CoA and lactonization to generate methylated alkylpyrones. The ability of PKS11 to select different extenders at different stages of polyketide elongation is intriguing, and more structural studies in this aspect will be useful for the generation of clinically important unnatural polyketides. 


\subsubsection{Pyrone Synthases from S. coelicolor}

Two pyrone synthases from S. coelicolor were recently characterized. Germicidin synthase (GCS) utilizes short to medium-chain acyl-CoAs (C4-C8) and acetoacetyl-CoA as starter units and malonyl-CoA, methylmalonyl-CoA and ethylmalonyl-CoA as extender units to generate various germicidins (42, Scheme 15) [74].

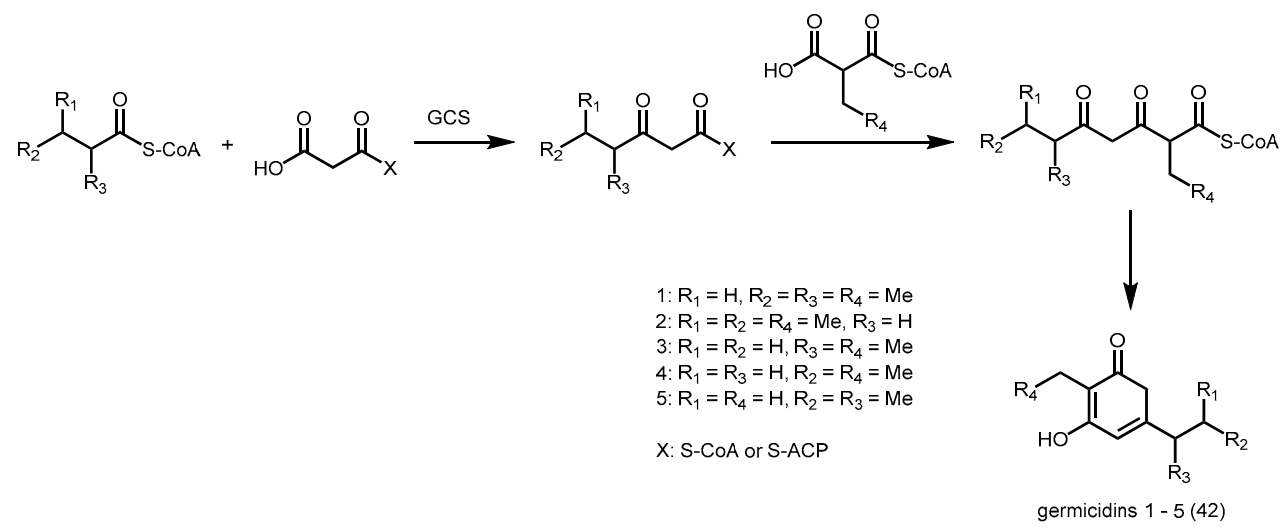

Scheme 15. Synthesis of various germicidins catalyzed by S. coelicolor GCS.

A type III polyketide synthase in S. coelicolor (locus tag: SCO7671) efficiently utilizes benzoyl-CoA and the branched iso-butyryl-CoA as starters to form pyrones. In addition, SCO7671 and GCS condensed malonyl-CoA with hexanoyl-ACP to yield pyrones; this is the first time type III PKSs were found to utilize acyl-ACPs $[74,75]$. Both PKSs are capable of accommodating huge macromolecular substrates as acyl carriers, presenting a novel means to manipulate PKSs to expand their repertoire of biosynthesized products. The crystal structure of GCS was recently solved, but more structural studies are needed before the molecular basis of the interaction between GCS and ACP can be understood [76].

\subsubsection{Other Bacterial Type III PKSs}

Recently, a heptaketide pyrone synthase from the soil bacterium Rhizobium etli (RePKS) was characterized. Unlike the R. palmatum aloesone synthase (ALS), RePKS undergoes dual cyclization reactions and generates a heptaketide pyrone 43 from one unit of acetyl-CoA and six units of malonyl-CoA (Scheme 16) [77]. RePKS also showed broad substrate specificities, generating triketide and/or tetraketide pyrones from acyl-CoAs (C4-C18) and benzoyl-CoA. Homology modeling highlighted that the active site cavity of RePKS is slightly larger than the active site cavity of THNS, hence partially explaining why RePKS can carry out more rounds of polyketide extension. More studies will be needed in order to provide insights into the differences in the mechanisms of action between RePKS, THNS, and R. palmatum ALS.

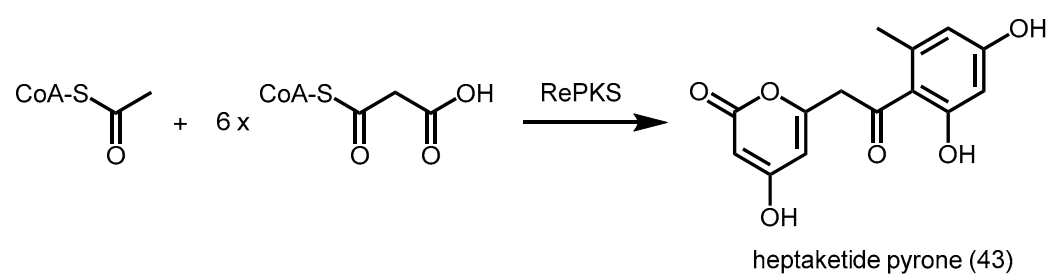

Scheme 16. Synthesis of heptaketide pyrone from R. etli RePKS.

In Azotobacter vinelandii, two alkylresorcinol synthases are encoded in the ars operon-ArsB and ArsC. They share $71 \%$ identity with each other but are enzymatically distinct PKSs. Both enzymes can utilize the same long-chain acyl-CoA as a starter and catalyze three successive decarboxylative condensations of malonyl-CoA to yield a tetraketide intermediate. Subtle differences in the active site 
residues of ArsB result in an intramolecular C2 to $C 7$ aldol condensation of the intermediate, followed by a decarboxylation to produce alkylresorcinols 44 (Scheme 17), which are vital components of the cyst coat that is generated during bacterial dormancy. However for ArsC, the intermediate undergoes a C5-oxygen to $\mathrm{C} 1$ lactonization to form alkylpyrones (Scheme 17) [78]. ArsB was found to be able to accept a variety of acyl-CoA starters (C10-C22) while ArsC showed a wider substrate range (C6 to C22).

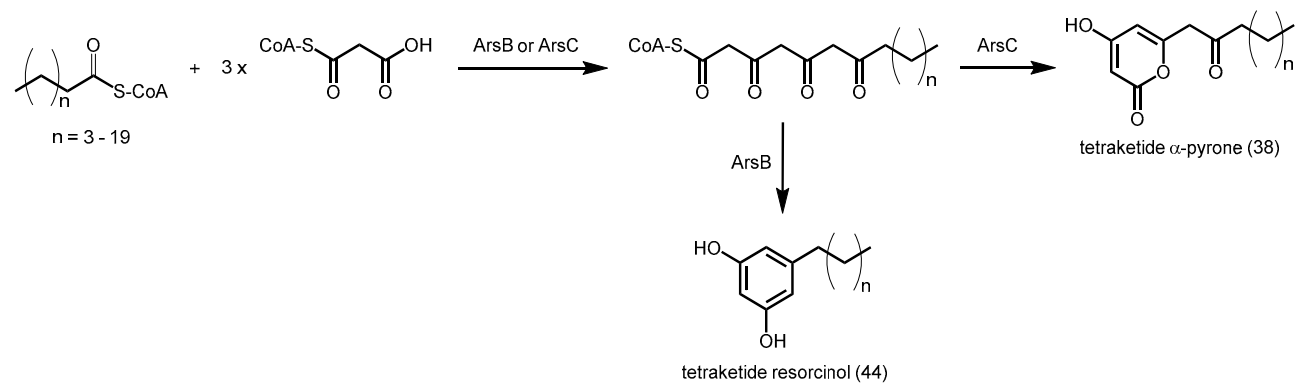

Scheme 17. Synthesis of tetraketide $\alpha$-pyrones and tetraketide resorcinols catalyzed by $A$. vinelandii ArsC and ArsB respectively.

In addition to straight chain acyl-CoAs, fatty acids bound to the ACP domain of the fatty acid synthase, ArsA, were found to be able to be directly transferred to the catalytic cysteines of ArsB and ArsC, indicating that unlike most type III PKSs, these PKSs can also utilize acyl-ACP as starters [79]. The crystal structure of ArsC (PDB ID: 3VS8) showed a hydrophobic acyl-binding tunnel similar to PKS18, explaining the ability of ArsC to utilize long-chain acyl-CoAs. Interestingly, mutating the Trp281 residue in ArsB to the corresponding Gly residue in ArsC resulted in an ArsB W281G mutant that was unable to produce any alkylresorcinol, but gained the synthesis of alkylpyrones. In contrast, the corresponding G284W mutation in ArsC resulted in a decrease in alkylpyrone production and a concomitant increase in alkylresorcinol generation [80]. This highlighted the fact that Trp281 in ArsB and Gly284 in ArsC play an important role in determining the cyclization specificity of the PKS. The crystal structure of the ArsC G284W mutant showed a significant decrease in the active site cavity volume when compared to that of the wild-type ArsC. Hence, the presence of an aromatic residue in the active sites of ArsB and the ArsC G284W mutant is likely to provide a steric wall for the tetraketide intermediates to be folded into a suitable configuration for aldol condensation to occur. The presence of the tryptophan residue at position 284 resulted in a modulation of the position of Met293 into the active site, greatly decreasing the space for polyketide synthesis (Figure 13).

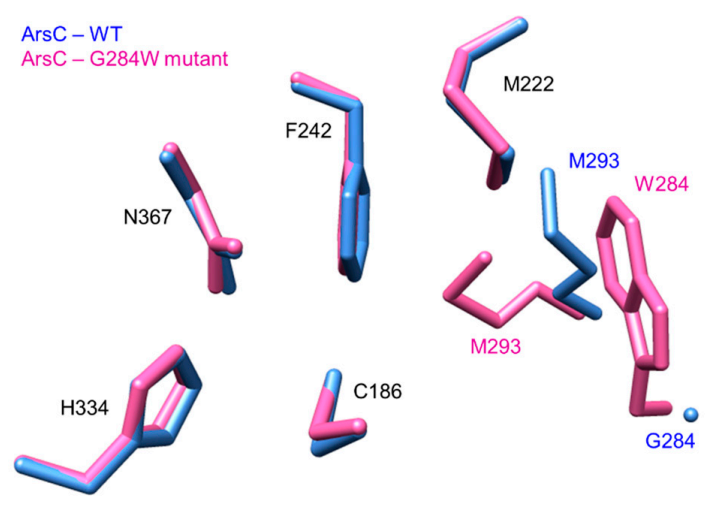

Figure 13. Active site cavity of ArsC-WT (blue, PDB ID: 3VS8) and ArsC-G284W mutant (pink, PDB ID: 3VS9). The Cys-His-Asn catalytic triad remained in their positions; the absence of tryptophan in position 284 allowed Met293 to swing out of the cavity, giving the growing polyketide more space. 
A type III PKS from Rhodospirillum centenum, Rhodospirillum polyketide synthase (RpsA), was characterized and found to utilize a variety of acyl-CoAs (C6-C20) as starters, followed by the decarboxylative condensations of one methylmalonyl-CoA, one malonyl-CoA, and another methylmalonyl-CoA to yield alkyldimethylpyrones 45 and/or alkyldimethylresorcylic acids 46 (Scheme 18) [81]. RpsA is the first bacterial type III PKS capable of accepting two units of methylmalonyl-CoA as extenders efficiently. More studies are needed to provide insights into the ability of RpsA to selectively control the condensation order of extenders at different stages of polyketide elongation.

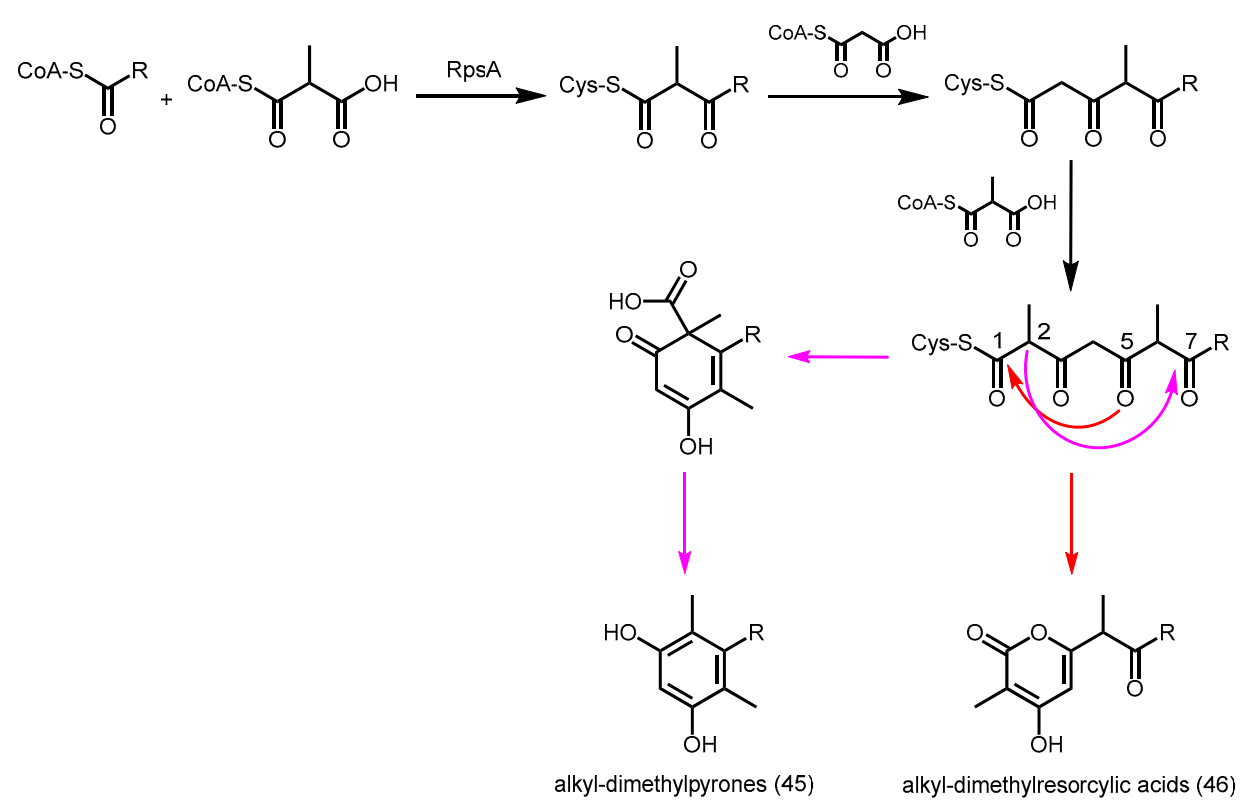

Scheme 18. Synthesis of alkyl-dimethylpyrones and alkyl-dimethylresorcylic acids by R. centenum RpsA.

\subsection{Fungal Type III PKSs}

Although most fungal polyketides such as lovastatin are synthesized by the iterative type I PKSs, fungal type III PKS genes were identified recently in the past decade. Using the alfalfa CHS amino acid sequence as a reference, four putative type III PKSs, namely chalcone synthase-like A, chalcone synthase-like B, chalcone synthase-like C, and chalcone synthase-like D (CsyA, CsyB, CsyC, and CsyD), were found in the filamentous fungus, Aspergillus oryzae. Similar CHS-like genes were also identified in Fusarium graminearum and Neurospora crassa (one homolog each), Magnaporthe grisea and Podospora anserina (two homologs each), Phanerochaete chrysosporium (three homologs each) [19], and several species of lichen fungi [82].

The first fungal type III PKS was characterized from the filamentous fungus, N. crassa, and showed approximately $26 \%$ identity to other plant and bacterial PKSs [83]. 2'-oxoalkylresorcylic acid synthase (ORAS) from N. crassa was found to catalyze the decarboxylative condensation of four units of malonyl-CoA with a unit of long-chain acyl-CoA (C18) as the preferred starter, followed by a $\mathrm{C} 2$ to C7 aldol condensation to generate stearoyl pentaketide resorcylic acid (47) (Scheme 19) [20].

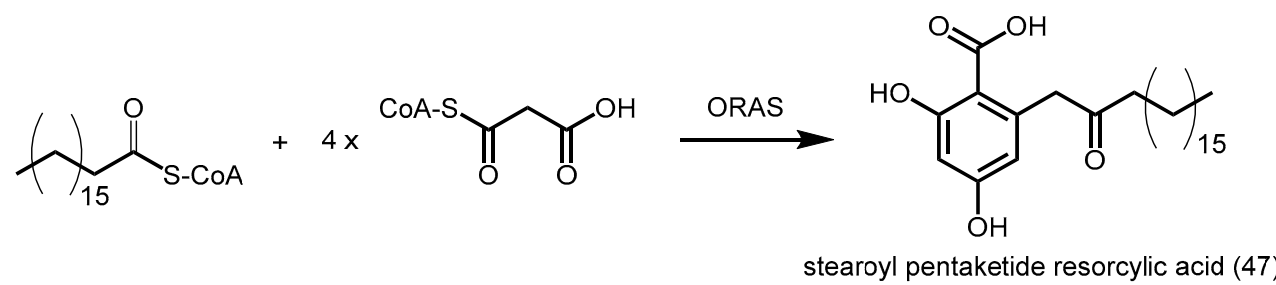

Scheme 19. Synthesis of stearoyl pentaketide resorcylic acid catalyzed by N. crassa ORAS. 
ORAS was also capable of accepting a range of acyl-CoAs (C2-C24) as starters to produce triketide alkylpyrones (from C2 to C20 acyl-CoA substrates), tetraketide alkylpyrones (from C8-C16 acyl-CoA substrates), tetraketide alkylresorcinols (from C10-C24 acyl-CoA substrates), tetraketide alkylresorcylic acids (from C20 acyl-CoA substrate), pentaketide alkylresorcinols (from C16-C20 acyl-CoA substrates), and pentaketide alkylresorcylic acids (from C18-C20 acyl-CoA substrates). Similar to most bacterial type III PKSs, ORAS contains a second active site thiol (Cys120) at a position corresponding to the THNS Cys106, which may help in steering the polyketide reactivity [84]. The crystal structure of ORAS (PDB ID: 3EUT) also bears structural resemblance to PKS18 and E. siluculosus phloroglucinol synthase (PhlD), and has a deep hydrophobic acyl-binding tunnel for accommodating long-chain acyl-CoAs, along with the conserved catalytic triad (Cys152, His305, and Asn338) and the CoA-binding tunnel (Figure 14) [83]. However, several critical changes in the active site residues result in a different product chemistry for ORAS, and more studies are needed in order to better understand the mechanism of action of ORAS.

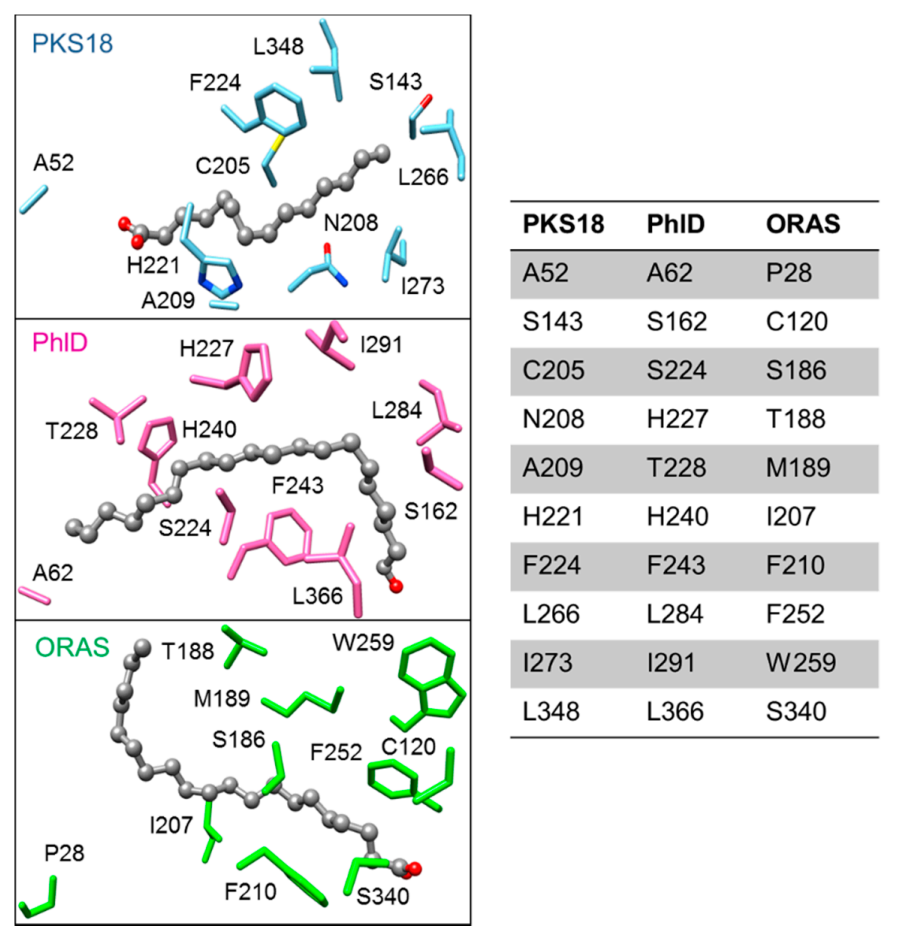

Figure 14. Acyl-binding tunnel of the following type III PKSs: M. tuberculosis PKS18 (blue, PDB ID: 1TED), E. siliculosus PhID (pink, PDB ID: 4B0N), and N. crassa ORAS (green, PDB ID: 3EUT). The table shows the residues lining the acyl-binding tunnel of the respective PKSs. PKS18 and PhID are more similar compared to ORAS; it is possible that these changes lining the acyl-binding tunnel lead to a different product chemistry in ORAS compared to PKS18 and PhID.

Both A. oryzae CsyA and CsyB showed 38\% identity with ORAS [85]. CsyA is likely to be a pyrone synthase (Scheme 20), capable of utilizing C4-C18 acyl-CoAs with malonyl-CoA as the extender to generate triketide pyrones. Furthermore, reactions with longer starters (C10-C18) resulted in the production of tetraketide pyrones.

CsyB is the first type III PKS capable of catalyzing the condensation of two diketide CoA esters, followed by cyclization to yield pyrones. CsyB was found to utilize acetoacetyl-CoA (48) both as a starter and as an extender to form dehydroacetic acid (49) (Scheme 21A) [86]. Similarly, two units of $\beta$-ketohexanoyl-CoA (50) can be coupled together by CsyB to form 3-butanoyl-4-hydroxy-6-propyl$\alpha$-pyrone (51) (Scheme 21B). Furthermore, CsyB is able to catalyze a single decarboxylative condensation of malonyl-CoA with C4-C10 acyl-CoAs to produce a $\beta$-keto fatty acyl intermediate, which can then undergo a second condensation with acetoacetyl-CoA to form 3-acetyl-4-hydroxy- 6-alkyl- $\alpha$-pyrones. 
If acetoacetyl-CoA is unavailable, CsyB is also able to catalyze the condensation of malonyl-CoA with acetyl-CoA to generate acetoacetyl-CoA (Scheme 21A). The broad substrate specificities and unique coupling reaction mechanisms of $\mathrm{CsyB}$ make it an ideal target for future structural studies.

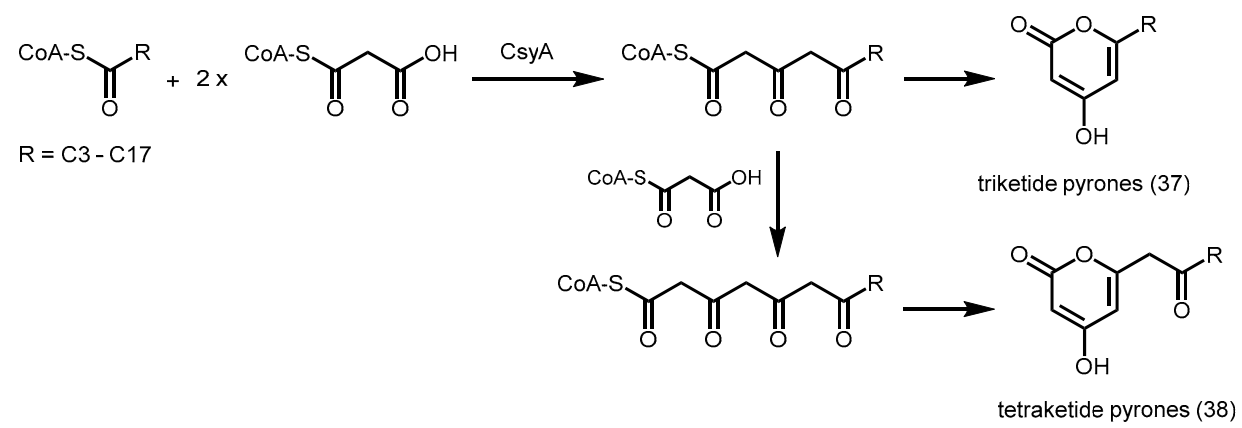

Scheme 20. Synthesis of triketide and tetraketide pyrones catalyzed by A. oryzae CsyA.

(A)

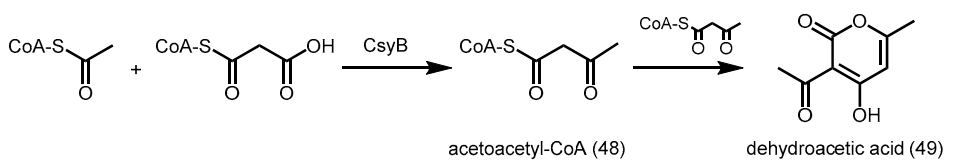

(B)

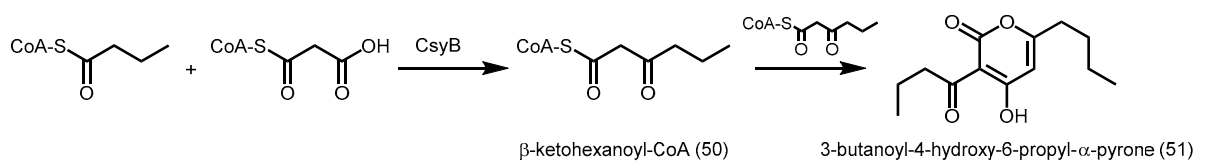

Scheme 21. Synthesis of (A) dehydroacetic acid and (B) 3-butanoyl-4-hydroxy-6-propyl- $\alpha$-pyrone catalyzed by A. oryzae CsyB.

An unusual type III PKS with extremely broad substrate specificity was cloned from Aspergillus niger. Aspergillus niger polyketide synthase (AnPKS) shares 37\% identity with ORAS, and homology modeling using the structure of ORAS as a reference reveals a similar acyl-binding tunnel [21]. Indeed, AnPKS was found to utilize a variety of acyl-CoAs (C2-C18) as starters and malonyl-CoA as the extender to generate triketide alkylpyrones. AnPKS was also able to form tetraketide alkylpyrones, tetraketide alkylresorcinols, and pentaketide alkylresorcinols $\mathbf{5 3}$ from C10-C18 acyl-CoAs (Figure 15A). Furthermore, acetoacetyl-CoA, the aromatic acyl-CoAs such as benzoyl-CoA and phenylacetyl-CoA (54), and branched acyl-CoAs such as isobutyryl-CoA (55), isovaleryl-CoA (56), and methylcrotonyl-CoA (57), are readily accepted by AnPKS (Figure 15B). The promiscuity of AnPKS makes it an ideal target for the engineered biosynthesis of unnatural polyketides with potential drug applications.

(A) Products formed catalyzed by AnPKS and/or BPKS

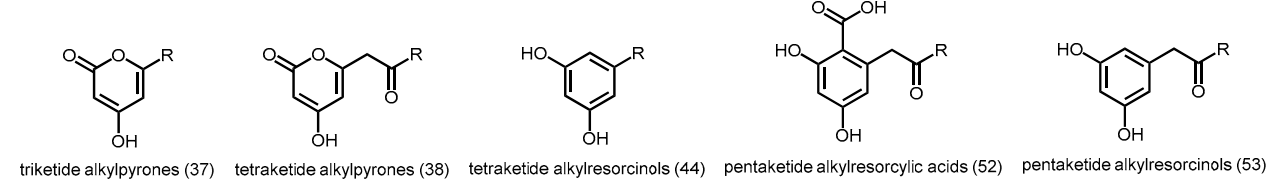

(B) Starter acyl-CoA units accepted by AnPKS and/or BPKS

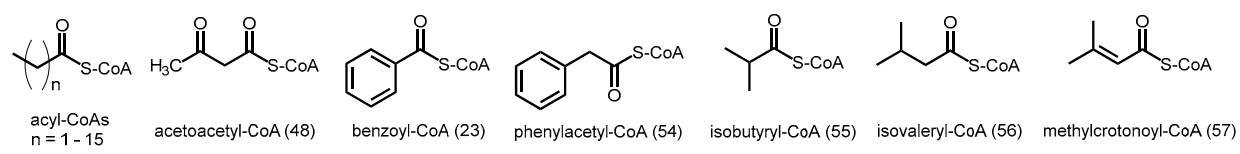

Figure 15. Substrate and product profiles of A. niger AnPKS and B. cinerea BPKS. (A) Products formed, and (B) starter acyl-CoAs accepted by the enzyme. 
The Botrytis cinerea type III PKS (BPKS) was also recently found to accept short-chain to long-chain acyl-CoAs (C2-C18) as starters with malonyl-CoA as the extender to generate triketide alkylpyrones (Figure 15A) [87]. Tetraketide alkylpyrones were also formed from C10-C18 acyl-CoAs, while pentaketide alkylresorcylic acids $\mathbf{5 2}$ and pentaketide alkylresorcinols $\mathbf{5 3}$ were also produced from C16-C18 acyl-CoAs. Moreover, BPKS is able to generate a hexaketide alkylresorcylic acid from C18 acyl-CoA. BPKS is also able to utilize acetoacetyl-CoA and the aromatic benzoyl-CoA as starters to form triketide pyrones (Figure 15B). BPKS shares 65\% identity with ORAS and similar to other type III PKSs which utilize long-chain acyl-CoAs as substrates, homology modeling studies show an acyl-binding tunnel in the active site. Recently, pyrones have been found to possess anti-cancer [88] and antibacterial [89] properties, making them potential lead structures for bioactive agents. However, more studies are needed to understand how differential chain extension and thus product profiles of BPKS and other fungal type III PKSs can be regulated based on the length of the starter units.

\section{Precursor-Directed Biosynthesis of Polyketides}

Although type III PKSs share a similar three-dimensional $\alpha \beta \alpha \beta \alpha$ fold and a conserved Cys-His-Asn catalytic triad, subtle differences in the active site cavities result in diverse substrate specificities and different product profiles. Notably, manipulation of the biosynthetic reaction by the incorporation of unnatural substrates has led to the generation of novel polyketide libraries. For instance when both the starters and the extenders were substituted with non-physiological substrates, CHS from the herb Scutellaria baicalensis was able to catalyze the formation of unnatural polyketides [90]. Other than the preferred substrates of $p$-coumaroyl-CoA and malonyl-CoA, S. baicalensis CHS was able to accept benzoyl-CoA as the starter and methylmalonyl-CoA as the extender to produce methylated triketide and tetraketide pyrones. Furthermore, the CHS also catalyzed the decarboxylative condensation of methylmalonyl-CoA with hexanoyl-CoA to yield a methylated triketide pyrone. CHSs from the Thai medicinal plant Cassia alata were also found to be promiscuous and accepted butyryl-CoA, isovaleryl-CoA, hexanoyl-CoA, benzoyl-CoA, cinnamoyl-CoA, and $p$-coumaroyl-CoA as starters to form triketide and tetraketide pyrones, and phloroglucinol derivatives in the presence of malonyl-CoA as the extender [91]. The ability of CHSs to utilize CoA esters that are not derived from the phenylpropanoid pathway highlights the role CHSs can play in synthesizing diverse bioactive polyketide compound libraries.

Similar to studies on CHSs, STS from Arachis hypogaea (peanut) was also found to accept several unnatural substrates to produce unique polyketides [92]. In particular, incubation of peanut STS with $p$-fluorocinnamoyl-CoA (58), trans-3-furanacryloyl-CoA (59), or trans-3-(3-thienyl)acryloyl-CoA (60, Figure 16) in the presence of malonyl-CoA yielded stilbene-like products.
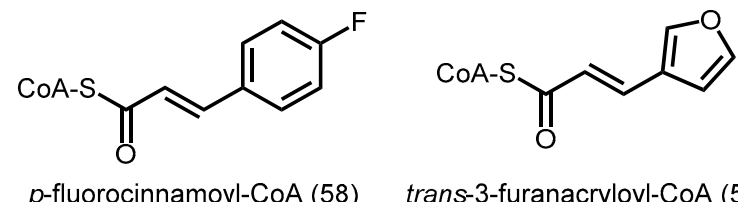

trans-3-furanacryloyl-CoA (59)

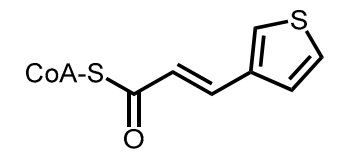

trans-3-(3-thienyl)acryloyl-CoA (60)

Figure 16. Starter acyl-CoA substrates utilized by A. hypogaea STS.

When the peanut STS was incubated with other $p$-substituted cinnamoyl-CoA analogues (-Cl, $-\mathrm{Br}$, and $\left.-\mathrm{OCH}_{3}\right)$, only triketide and tetraketide pyrones were obtained. This indicated potential steric effects by substituents that are larger than that of the wild-type substrate (-OH), which affected the stability and/or the conformation of polyketide intermediates in the cyclization pocket of the active site. Interestingly, when $p$-coumaroyl-CoA, cinnamoyl-CoA, or phenylpropionyl-CoA were utilized as starters, Scots pine STS was able to form pentaketide stilbenes in addition to tetraketide and triketide products, suggesting a considerable amount of flexibility in chain elongation in the active site cavity [93].

Octaketide synthase (OKS) from A. arborescens is known to catalyze seven sequential decarboxylative condensations of malonyl-CoA with an acetyl-CoA starter molecule to form the 
octaketides SEK4 (12) and SEK4b (13) (Scheme 2). Further studies on substrate tolerance revealed that aloe OKS is able to accept $p$-coumaroyl-CoA as a starter to generate a novel hexaketide stilbene (61) and heptaketide chalcone (63) in the presence of malonyl-CoA as the extender (Scheme 22) [94]. Similarly, OKS condenses malonyl-CoA with hexanoyl-CoA, followed by a C6 to C11 or C12 to C7 aldol-type cyclization to yield a hexaketide resorcinol (62) or heptaketide phloroglucinol (64) respectively (Scheme 22). The aloe OKS also utilizes long-chain C8-C20 acyl-CoAs as starters to form triketide and tetraketide $\alpha$-pyrones [40]. This indicates that the 'downward expanding' active site architecture of aloe OKS is large enough to accommodate bulky substrates and intermediates. A similar octaketide-producing PKS (HpPKS2) from Hypericum perforatum (St. John's wort) was also found to be promiscuous and accepted isobutyryl-CoA, hexanoyl-CoA, and benzoyl-CoA as starters to generate a series of triketide to heptaketide products [95].

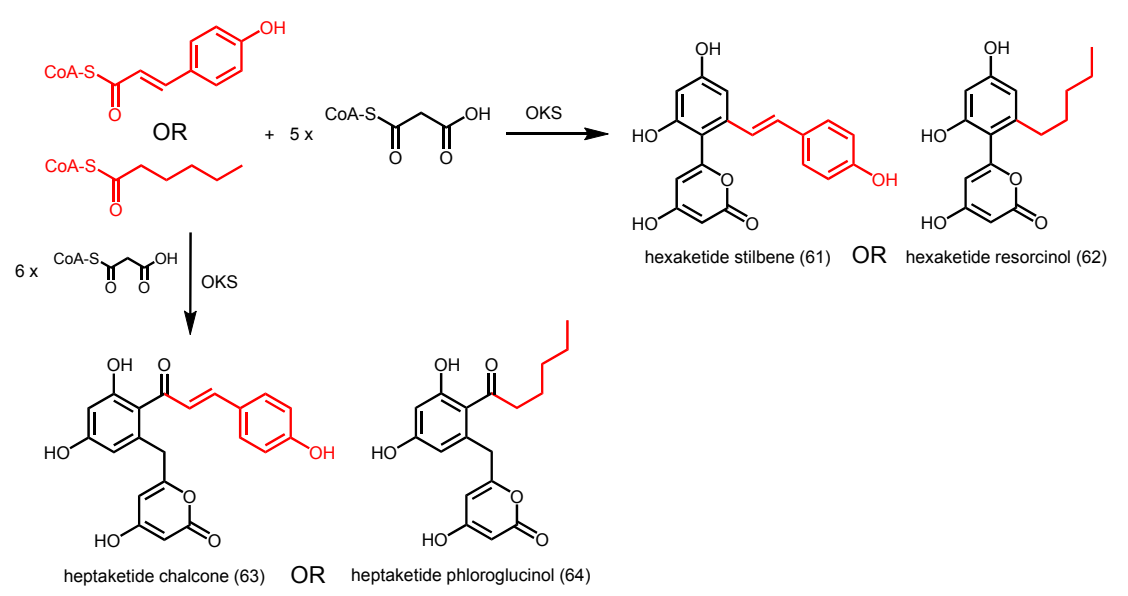

Scheme 22. Synthesis of various polyketides catalyzed by A. arborescens OKS.

The rhubarb BAS catalyzes a single decarboxylative condensation of malonyl-CoA to $p$-coumaroyl-CoA, followed by another decarboxylation to form the diketide benzalacetone. As a result of a wide active site entrance caused by a F215L replacement (numbering in alfalfa CHS) (Figure 6B), rhubarb BAS is able to accept a unique range of substrates, including the bulky $N$-methylanthraniloyl-CoA as a starter with malonyl-CoA or methylmalonyl-CoA as the extender to yield quinolone alkaloids [96,97]. Furthermore, the promiscuous enzyme is able to catalyze the decarboxylative condensation of malonyl-CoA with phenylalanyl-CoA (65), followed by intramolecular lactamization to form 2,4-pyrrolidinedione (66). 2,4-pyrrolidinedione and its analogs may undergo further spontaneous dimerization (Scheme 23) [97]. Interestingly, the tetramic acid dimer, $2^{\prime}, 5-1^{\prime}, 2^{\prime}$-dihydro-4-hydroxy-2',5-bis(phenylmethyl)-[3,3'-Bi-5Hpyrrole]-2,5'(1H)-dione (67), exhibited moderate cytotoxic activity against murine leukemia P388 cells, thus highlighting the utility of precursor-directed biosynthesis.

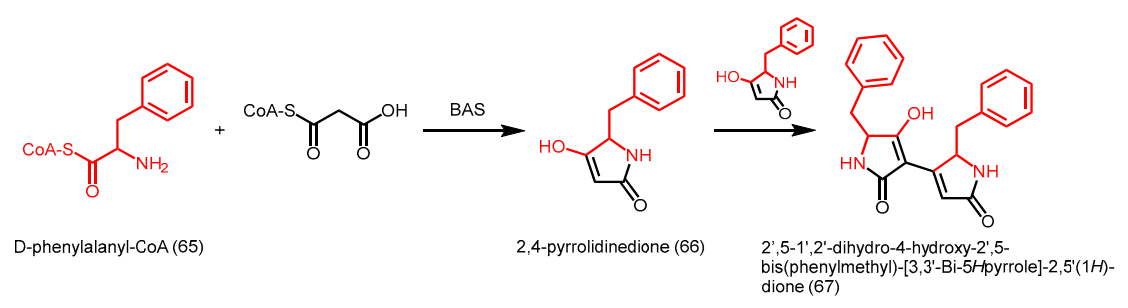

Scheme 23. Novel polyketides catalyzed by R. palmatum BAS.

Numerous studies have illustrated the feasibility of performing precursor-directed biosynthesis using type III PKSs to generate novel polyketides. Indeed, by establishing a combinatorial biosynthetic route in Escherichia coli [98] and exploring the substrate promiscuity of a mutant CHS with STS activity (18xCHS) from alfalfa [26], at least 413 potentially novel polyketide compounds were biosynthesized 
(manuscript in preparation). In this approach, various combinations of 69 starter acyl-CoA and 12 extender acyl-CoA precursors generated by several promiscuous acid-CoA ligases were delivered to the mutant CHS, and the substrate profile was established. Similar work in precursor-directed biosynthesis using a chalcone synthase from $O$. sativa led to the development of novel polyketides as antimicrobial drug leads [28]. The structural and chemical diversity of products make type III PKSs an excellent platform for the engineered biosynthesis of novel bioactive polyketides. More structural variants of starter and extender substrates can be tested in order to generate comprehensive libraries of compounds which may serve as potential drug leads.

\section{Insights into Mutagenesis Studies}

Crystallographic analyses of the plant type III PKSs (alfalfa CHS [23], Scots pine STS [26], daisy 2-PS [35], rhubarb BAS [45], O. sativa CUS [49], C. microcarpa ACS and QNS [59], and brown alga phloroglucinol synthase [60]), the bacterial PKSs (S. coelicolor THNS [66], M. tuberculosis PKS11 [73] and PKS18 [72], A. vinelandii ArsC [80]), and of the fungal ORAS from N. crassa [83] have enabled the understanding of the basis of starter molecule selectivity, chain elongation, and cyclization of the linear polyketide intermediate. These structural studies have provided insights into the mechanistic details of type III PKSs, illustrating how subtle modifications in the active site cavity can expand the biosynthetic repertoire and result in the generation of diverse products. Manipulation of active site residues by random mutagenesis [68] and/or site-directed mutagenesis has proved useful in the understanding of and subsequently, engineering of the substrate specificity and activity of type III PKSs.

\subsection{Dissection of Catalytic and Structural Roles of Residues via Mutagenesis}

The active site of alfalfa CHS (PDB ID: 1CGK) was found to be situated at the intersection of a CoA-binding tunnel and a bi-lobed cavity, which comprises a coumaroyl-binding pocket and a cyclization pocket [27]. Site-directed mutagenesis of the catalytic Cys164 to alanine, serine, or aspartate resulted in no naringenin production, confirming the role of Cys164 as the nucleophile and attachment site for the starter unit [25]. Mutations of Cys164 only modestly affected the malonyl-CoA decarboxylation activity (C164A and C164S), whereas mutations of Phe215, His303, and Asn336 abolished (H303A, H303D, H303N, H303T, N336H, and N336Q) or severely impaired (F215S, F215W, F215Y, N336A, N336D, and N3336K) malonyl-CoA decarboxylation activities, except for the isosteric mutation of His303 to a glutamine. Hence, Cys164 is not important for malonyl-CoA decarboxylation and replacing the nucleophile with a neutral alanine residue only prevents the loading of the starter molecule. The ability of His303 to hydrogen bond with the thioester carbonyl oxygen of the extender substrate and stabilize the negative charge in the acetyl carbanion was found to be essential for malonyl-CoA decarboxylation (Figure 17). In addition, Cys164, Phe215, and His303 mutants did not significantly affect the $\mathrm{K}_{\mathrm{d}}$ values of CoA or acetyl-CoA, suggesting that these residues do not contribute to binding of CoA esters in the active site. Although the binding of CoA was not considerably affected, all Asn336 mutants except $\mathrm{N} 336 \mathrm{H}$ showed a 15-40 fold increase in $\mathrm{K}_{\mathrm{d}}$ values for acetyl-CoA binding compared to wild-type CHS, thus indicating that this residue contributes to the binding of substrates with a thioester carbonyl. The hydrogen bond between Asn336 and the thioester carbonyl oxygen of malonyl-CoA serves as a guide to ensure the proper conformation for interaction with His303, and for decarboxylation and subsequent condensation of the acetyl carbanion to the enzyme-bound starter unit or polyketide intermediate (Figure 17). Similarly, Phe215 may orient the terminal carboxylate of the extender via van der Waals forces, and aid in malonyl-CoA decarboxylation and chain extension of the polyketide intermediate.

Mutagenesis studies have also identified various non-catalytic residues lining the active site cavity that are believed to be important in determining the chain length specificity and the substrate and product profiles of PKSs. These residues include Thr132, Ser133, Thr194, Thr197, Gly256, Phe265, and Ser338 (numbering in alfalfa CHS) which are typically replaced in other type III PKSs but are conserved in CHSs [27]. Daisy 2-pyrone synthase (2-PS) shares the same overall structure and catalytic 
triad as alfalfa CHS, but variations in three residues (T197L, G256L, and S338I) lining the active site cavity results in a significant reduction in the active site volume [35]. The 2-PS structure was the first to demonstrate a simple means of functional divergence in type III PKSs, whereby steric restriction could alter the selectivity of the starter molecule, the number of chain elongation reactions, and the cyclization chemistry employed to terminate and release the polyketide product.

(A) Decarboxylation of malonyl-CoA

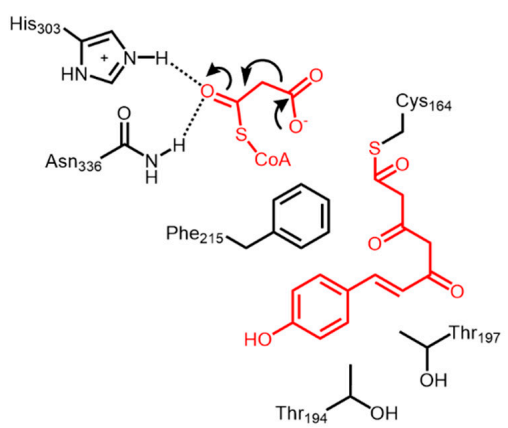

(B) Condensation of acetyl carbanion with polyketide intermediate

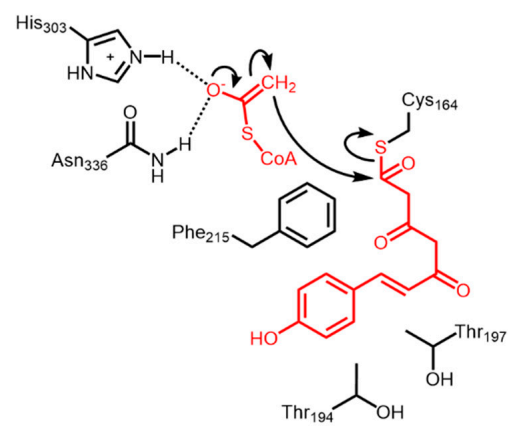

Figure 17. (A) Decarboxylation of the extender malonyl-CoA is catalyzed by His303 and Asn336 in alfalfa CHS. Phe215 is thought to orient the terminal carboxylate of malonyl-CoA and aid in the reaction; (B) The acetyl carbanion subsequently undergoes a condensation with the enzyme-bound triketide intermediate.

In H. androsaemum benzophenone synthase (BPS), a T132L mutation (numbering in alfalfa CHS) resulted in changes in the active site cavity which limited polyketide chain extension and transformed the enzyme from a biphenyl synthase into a phenylpyrone synthase with a trace of the wild-type activity [99]. In another study, structure-based mutagenic transformation of alfalfa CHS resulted in a change in chain termination chemistry from a Claisen condensation typical of CHSs to an aldol condensation usually catalyzed by STSs, indicating that functional divergence can be determined by electronic factors rather than steric effects of the active site cavity [26].

Aloe contains two highly similar type III PKSs, pentaketide chromone synthase (PCS) and octaketide synthase (OKS), which share more than $90 \%$ identity with each other. Although these two enzymes possess different biochemical functions, a single mutational change of Met197 in the pentaketide-producing PCS to Gly197 in OKS (numbering in alfalfa CHS) resulted in the synthesis of the octaketides, SEK4 (12) and SEK4b (13) (Scheme 2) [38]. Conversely, a reciprocal mutation in aloe OKS to the bulky methionine residue resulted in a loss of octaketide synthase activity and a gain in pentaketide synthase activity [40]. In addition, various small-to-large substitutions in OKS (G197A, G197T, G197M, G197L, G197F, and G197W) resulted in a loss of octaketide production and a concomitant generation of shorter chain length polyketides (from heptaketide to triketide), depending on the steric bulk of the side chain. Therefore, residue 197 is likely to control polyketide chain length and product specificity. The reduced steric bulk due to the M197G substitution in the PCS mutant unlocks two buried pockets, thus expanding the available cyclization cavity to accommodate the octaketide product [38]. Interestingly, the location and orientation of one of the pockets of the PCS M197G mutant superimpose well with a similar acyl-binding tunnel in OKS [94], in bacterial type III PKSs such as THNS [66] and PKS18 [72], and in the fungal 2'-oxoalkylresorcylic acid synthase (ORAS) [83]. Since aloe OKS can utilize long-chain (C6-C20) acyl-CoAs to form triketide and tetraketide pyrones [40], it is possible that the plant, bacterial, and fungal type III PKSs share a similar active site architectural strategy to synthesize the long-chain polyketides such as pyrones, phloroglucinols, and resorcinols.

\subsection{Structure-Based Engineering and the Versatility of Type III PKSs}

Manipulation of the type III PKS reactions by using rationally engineered mutant enzymes in conjunction with substrate analogues can result in a further generation of chemically and structurally 
distinct unnatural polyketides. The 'gatekeeper' Phe215 is generally conserved in most type III PKSs and is located between the CoA-binding tunnel and the entrance of the active site cavity. Despite having a lower malonyl-CoA decarboxylation activity compared to wild-type alfalfa CHS, the F215S mutant is able to utilize $p$-coumaroyl-CoA, phenylacetyl-CoA, benzoyl-CoA, and C5-C8 acyl-CoAs with less than $3 \%$ the catalytic efficiency of the wild-type enzyme to produce tetraketide lactones [100]. Intriguingly, although $N$-methylanthraniloyl-CoA is not normally utilized by the wild type CHS, the F215S mutant was found to accept the bulky starter with a comparable $k_{\text {cat }} / \mathrm{K}_{\mathrm{m}}$ to $R$. graveolens acridone synthase (ACS), to form a tetraketide $N$-methylanthraniloyltriacetic acid lactone (31). A modification into a serine residue at position 215 results in a reduction in steric hindrance and a wider active site entrance, thus allowing the entry of the bulky $N$-methylanthraniloyl-CoA.

Although octaketides are not usually synthesized by the wild type S. baicalensis CHS, the S338V mutant CHS was found to produce trace amounts of SEK4 (12) and SEK4b (13) using eight units of malonyl-CoA (Scheme 2C) [101]. This S338V mutation was proposed to facilitate the extension of the linear polyketide intermediate into a buried pocket in the active site cavity of the enzyme by providing steric guidance. It was demonstrated for the first time that CHS could be engineered to generate longer polyketides such as octaketides. Furthermore, the synthesis of SEK4 (12) and SEK4b (13) by S. baicalensis CHS was dramatically improved in an OKS-like triple mutant (T197G/G256L/S338V). These results suggested that rational site-directed mutagenesis efforts can be used to generate CHSs with different substrate selectivities and altered product profiles, thereby producing diverse novel polyketides.

Structure-based engineering efforts on the aloe PCS and OKS further extended the biosynthetic repertoire of type III PKSs. When two aromatic residues at the bottom of the buried pocket of the PCS M207G mutant (corresponding to Thr197 in alfalfa CHS) were simultaneously replaced by alanine, the active site cavity was further expanded by approximately four times compared to the wild-type PCS [102]. Interestingly, the PCS F80A/Y82A/M207G mutant was able to catalyze the decarboxylative condensation of nine units of malonyl-CoA with an altered mechanism of cyclization to yield an unnatural nonaketide naphthopyrone (68) (Scheme 24A). Similarly in OKS, when Asn222 situated at the bottom of the elongation cavity was replaced by a glycine residue, a novel decaketide benzophenone SEK15 (69) was produced from ten units of malonyl-CoA (Scheme 24B) [94]. The N222G mutant OKS was also able to accept phenylacetyl-CoA or benzoyl-CoA as starters to form novel octaketides and heptaketide benzophenone respectively [103]. Simultaneous mutation of the neighbouring Phe66 residue to leucine resulted in an OKS double mutant that is capable of synthesizing an unnatural dodecaketide naphthophenone TW95a (70) by the successive decarboxylative condensations of 12 units of malonyl-CoA, making it one of the longest polyketide synthesized by the structurally simple type III PKS (Scheme 24C) [104].

(A)

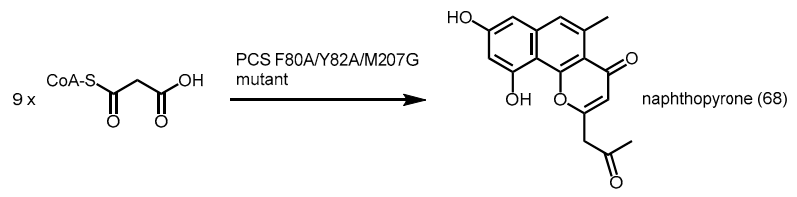

(B)

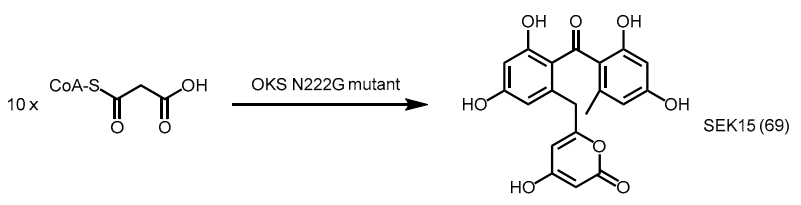

(C)

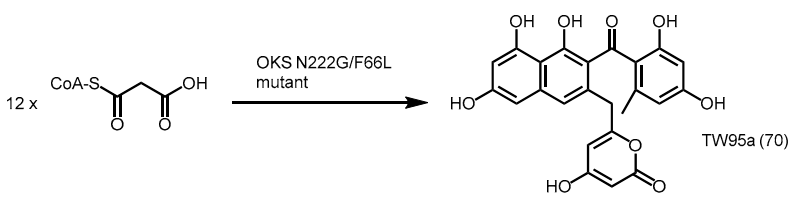

Scheme 24. Novel polyketides biosynthesized by mutant type III PKSs. (A) PCS F80A/Y82A/M207G mutant catalyzes the formation of napthopyrone; (B) OKS N222G mutant catalyzes the formation of SEK15; and (C) OKS N222G/F66L mutant catalyzes the formation of TW95a. 
A recently discovered CHS from a primitive club moss Huperzia serrata (HsPKS1) was found to display unusually broad substrate selectivity and produced unnatural polyketide-alkaloid hybrid molecules [105]. Using precursor-directed approaches, HsPKS1 synthesized a novel pyridoisoindole (74) from the condensation of 2-carbamoylbenzoyl-CoA (71), a synthetic nitrogen-containing non-physiological starter, with two units of malonyl-CoA (Scheme 25A). Conversely, the promiscuous aloe OKS was only able to generate a tetraketide pyrone as a minor product from the bulky synthetic starter. HsPKS1 was also able to generate novel alkaloids from the starters, 3-carbamoylpicolinoyl-CoA (72) and 3-carbamoyl-2-naphthoyl-CoA (73) (Scheme 25A). The structure-based S348G mutant (corresponding to Ser338 in alfalfa CHS) results in an expansion of the active site cavity which not only extended the polyketide chain length, but also altered the cyclization mechanism to yield a biologically active, ring-expanded dibenzoazepine (75) from the condensation of 2-carbamoylbenzoyl-CoA with three units of malonyl-CoA (Scheme 25B).

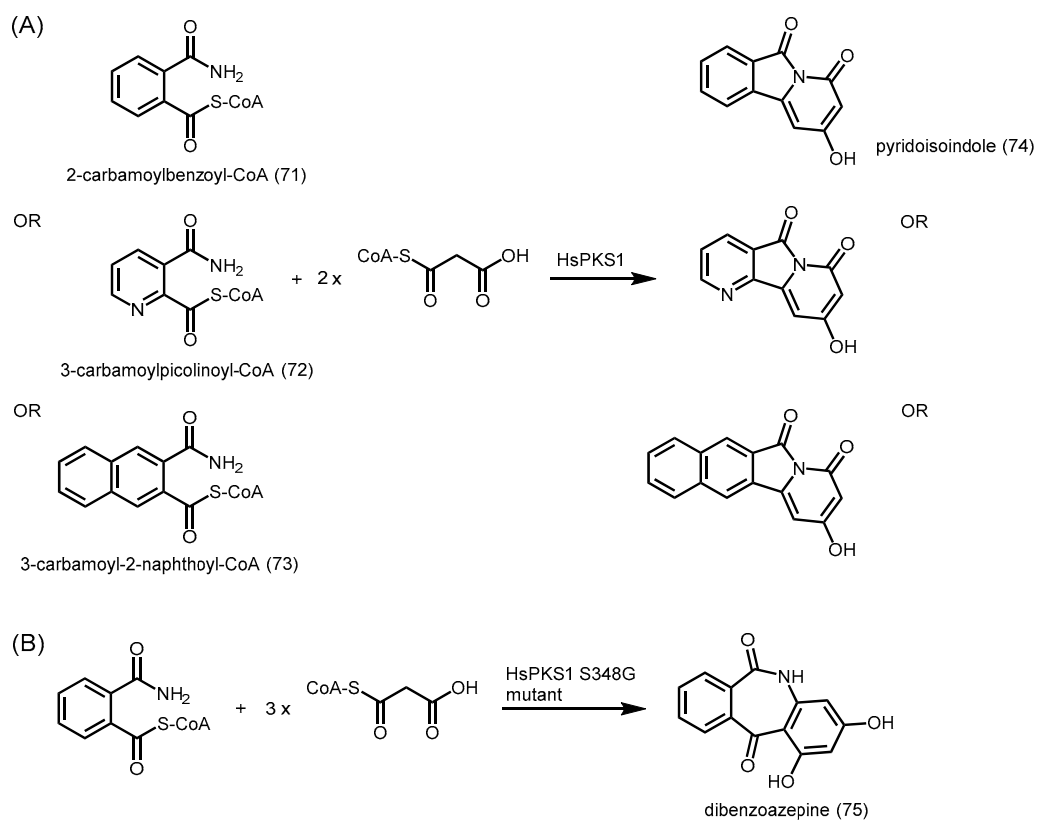

Scheme 25. Synthesis of polyketide-alkaloid hybrids catalyzed by (A) H. serrata HsPKS1 and its (B) S348G mutant.

Remarkably, the dibenzoazepine alkaloid inhibited biofilm formation by methicillin-susceptible Staphylococcus aureus (MIC $=12.0 \mu \mathrm{g} / \mathrm{mL}$ ). Hence, structure-based engineering of type III PKSs coupled with precursor-directed biosynthesis using rationally designed substrate analogues can provide an excellent platform for the further development of unnatural novel polyketides with unique therapeutic functions.

Studies on S. griseus RppA showed that the bulky Tyr224 (corresponding to Gly256 in alfalfa CHS) is important for RppA to select malonyl-CoA as a starter unit [106]. Mutations at this site (Y224A, Y224C, Y224G, and Y224L) led to a decrease in competition for malonyl-CoA and the mutant enzyme was able to utilize phenylacetyl-CoA or hexanoyl-CoA instead to synthesize tetraketide and triketide pyrones. This is analogous to the G256L replacement in rhubarb ALS which utilizes acetyl-CoA as a starter instead of the bulky $p$-coumaroyl-CoA, suggesting that the residue at position 256 controls starter unit selectivity [37]. Similar Tyr224 mutations in S. coelicolor THNS showed that the mutant enzyme can produce triketide pyrones from acetyl-CoA, acetoacetyl-CoA, hexanoyl-CoA, and benzoyl-CoA starters in significantly higher amounts compared to the wild-type THNS, due to a decrease in loading efficiency of the malonyl-CoA starter [107]. In addition, Ala305 (corresponding to Ser338 in alfalfa CHS) was found to be important for chain elongation, as a bulky 2-PS-like A305I 
mutation in S. griseus RppA resulted in the generation of triketide pyrones only from phenylacetyl-CoA or hexanoyl-CoA starters [106]. These results indicated that the overall backbone architecture of the active site cavity of RppA is similar to that of plant type III PKSs, thus structure-based engineering efforts in plant PKSs are likely to translate well to bacterial type III PKSs with minor differences.

\section{Conclusions}

Recently identified type III PKSs that possess novel catalytic activities, such as quinolone synthase (QNS), Rhodospirillum polyketide synthase (RpsA), and Botrytis cinerea type III PKS (BPKS), can provide unique polyketide scaffolds for synthetic enzymology. Until recently, biosynthesis of novel polyketide libraries has been dependent upon the broad substrate specificities of the type III PKSs. By rationally incorporating amino acid substitutions, the active site cavity of type III PKSs can be altered or expanded to facilitate the synthesis of more complex polyketides. This characteristic makes type III PKSs great candidates for structure-based engineering approaches to expand the chemical and structural diversity of natural products derived from these systems. Further studies on the catalytic potential and versatility of the functionally divergent type III PKSs should provide more intimate structural details of the biosynthetic processes.

Since the diversity of the polyketides biosynthesized by PKSs is partly dependent on the starter and extender units, variations in the choice of starters and extenders is important. However, there has been a lack of a toolkit that describes the means to introduce novel acyl-CoA precursors to PKSs for polyketide biosynthesis. Instead of chemical synthesis, an alternative way to generate a wide range of novel acyl-CoA esters is to make use of promiscuous acid-CoA ligases with unique carboxylic acid scaffolds [98]. By determining substrate promiscuities of acid-CoA ligases and PKSs beyond conventional substrate pools, we can establish novel enzymatic routes for the biosynthesis of unnatural polyketides which can be subsequently applied to drug screening efforts. These studies serve as a primer towards the development of novel and valuable polyketides with therapeutic potential via synthetic enzymology.

Acknowledgments: Molecular graphics and analyses were performed with the UCSF Chimera package. Chimera is developed by the Resource for Biocomputing, Visualization, and Informatics at the University of California, San Francisco (supported by NIGMS P41-GM103311). This review was supported by grants from the National Medical Research Council, Singapore, and the Academic Research Fund of the Ministry of Education, Singapore.

Author Contributions: The manuscript was written through contributions of all authors. All authors have given approval to the final version of the manuscript.

Conflicts of Interest: The authors declare no conflict of interest. The founding sponsors had no role in the design of the study; in the collection, analyses, or interpretation of data; in the writing of the manuscript, and in the decision to publish the results.

\section{Abbreviations}

The following abbreviations are used in this manuscript:

$\begin{array}{ll}\text { ACP } & \text { Acyl carrier protein } \\ \text { ACS } & \text { Acridone synthase } \\ \text { ALS } & \text { Aloesone synthase } \\ \text { AnPKS } & \text { Aspergillus niger polyketide synthase } \\ \text { ARAS2 } & \text { Alkyl-resorcylic acid synthase 2 } \\ \text { BAS } & \text { Benzalacetone synthase } \\ \text { BBS } & \text { Bibenzyl synthase } \\ \text { BIS } & \text { Biphenyl synthase } \\ \text { BPS } & \text { Benzophenone synthase } \\ \text { CHS } & \text { Chalcone synthase } \\ \text { CoA } & \text { Coenzyme A } \\ \text { CsyA } & \text { Chalcone synthase-like A } \\ \text { CTAL } & \text { Coumaroyl triacetic acid lactone }\end{array}$




$\begin{array}{ll}\text { CTAS } & \text {-coumaroyl triacetic acid synthase } \\ \text { CURS } & \text { Curcumin synthase } \\ \text { CUS } & \text { Curcuminoid synthase } \\ \text { DCS } & \text { Diketide-CoA synthase } \\ \text { DpgA } & \text { 3,5-dihydroxyphenylacetic acid synthase } \\ \text { GCS } & \text { Germicidin synthase } \\ \text { NAC } & \text { N-acetylcysteamine } \\ \text { OKS } & \text { Octaketide synthase } \\ \text { ORAS } & \text { 2'-oxoalkylresorcylic acid synthase } \\ \text { PCS } & \text { Pentaketide chromone synthase } \\ \text { PhlD } & \text { Phloroglucinol synthase } \\ \text { PKS } & \text { Polyketide synthase } \\ \text { PpASCL } & \text { Physcomitrella patens anther-specific chalcone synthase-like enzyme } \\ \text { 2-PS } & \text { 2-pyrone synthase } \\ \text { QNS } & \text { Quinolone synthase } \\ \text { STCS } & \text { Stilbenecarboxylate synthase } \\ \text { STS } & \text { Stilbene synthase } \\ \text { THNS } & \text { 1,3,6,8-tetrahydroxynaphthalene synthase }\end{array}$

\section{References}

1. Collie, J.N. Derivatives of the multiple keten group. J. Chem. Soc. Trans. 1907, 91, 1806-1813. [CrossRef]

2. Staunton, J.; Weissman, K.J. Polyketide biosynthesis: A millennium review. Nat. Prod. Rep. 2001, 18, 380-416. [CrossRef] [PubMed]

3. Calne, R.Y.; Collier, D.S.; Lim, S.; Pollard, S.G.; Samaan, A.; White, D.J.; Thiru, S. Rapamycin for immunosuppression in organ allografting. Lancet 1989, 2, 227. [CrossRef]

4. Alberts, A.W.; Chen, J.; Kuron, G.; Hunt, J.; Huff, J.; Hoffman, C.; Rothrock, J.; Lopez, M.; Joshua, H.; Harris, E.; et al. Mevinolin-A highly potent competitive inhibitor of hydroxymethylglutaryl-coenzyme a reductase and a cholesterol-lowering agent. Proc. Natl. Acad. Sci. USA 1980, 77, 3957-3961. [CrossRef] [PubMed]

5. Wright, L.F.; Hopwood, D.A. Actinorhodin is a chromosomally-determined antibiotic in Streptomyces coelicolor A3(2). J. Gen. Microbiol. 1976, 96, 289-297. [CrossRef] [PubMed]

6. Houtkooper, R.H.; Mouchiroud, L.; Ryu, D.; Moullan, N.; Katsyuba, E.; Knott, G.; Williams, R.W.; Auwerx, J. Mitonuclear protein imbalance as a conserved longevity mechanism. Nature 2013, 497, 451-457. [CrossRef] [PubMed]

7. Newman, D.J.; Cragg, G.M. Natural products as sources of new drugs over the 30 years from 1981 to 2010. J. Nat. Prod. 2012, 75, 311-335. [CrossRef] [PubMed]

8. Tatsuta, K.; Hosokawa, S. Total syntheses of polyketide-derived bioactive natural products. Chem. Rec. 2006, 6, 217-233. [CrossRef] [PubMed]

9. Birch, A.J.; Donovan, F.W. Studies in relation to biosynthesis. I. Some possible routes to derivatives of orcinol and phloroglucinol. Aust. J. Chem. 1953, 6, 360-368. [CrossRef]

10. Dunn, B.J.; Khosla, C. Engineering the acyltransferase substrate specificity of assembly line polyketide synthases. J. R. Soc. Interface R. Soc. 2013, 10, 20130297. [CrossRef] [PubMed]

11. Abe, I. Novel applications of plant polyketide synthases. Curr. Opin. Chem. Biol. 2012, 16, 179-185. [CrossRef] [PubMed]

12. Till, M.; Race, P.R. Progress challenges and opportunities for the re-engineering of trans-at polyketide synthases. Biotechnol. Lett. 2014, 36, 877-888. [CrossRef] [PubMed]

13. Shen, B. Polyketide biosynthesis beyond the type I, II and III polyketide synthase paradigms. Curr. Opin. Chem. Biol. 2003, 7, 285-295. [CrossRef]

14. Cox, R.J. Polyketides, proteins and genes in fungi: Programmed nano-machines begin to reveal their secrets. Org. Biomol. Chem. 2007, 5, 2010-2026. [CrossRef] [PubMed]

15. Stewart, C., Jr.; Vickery, C.R.; Burkart, M.D.; Noel, J.P. Confluence of structural and chemical biology: Plant polyketide synthases as biocatalysts for a bio-based future. Curr. Opin. Plant Biol. 2013, 16, 365-372. [CrossRef] [PubMed] 
16. Funa, N.; Ohnishi, Y.; Fujii, I.; Shibuya, M.; Ebizuka, Y.; Horinouchi, S. A new pathway for polyketide synthesis in microorganisms. Nature 1999, 400, 897-899. [PubMed]

17. Bangera, M.G.; Thomashow, L.S. Identification and characterization of a gene cluster for synthesis of the polyketide antibiotic 2,4-diacetylphloroglucinol from Pseudomonas fluorescens Q2-87. J. Bacteriol. 1999, 181, 3155-3163. [PubMed]

18. Pfeifer, V.; Nicholson, G.J.; Ries, J.; Recktenwald, J.; Schefer, A.B.; Shawky, R.M.; Schroder, J.; Wohlleben, W.; Pelzer, S. A polyketide synthase in glycopeptide biosynthesis: The biosynthesis of the non-proteinogenic amino acid (S)-3,5-dihydroxyphenylglycine. J. Biol. Chem. 2001, 276, 38370-38377. [CrossRef] [PubMed]

19. Seshime, Y.; Juvvadi, P.R.; Fujii, I.; Kitamoto, K. Discovery of a novel superfamily of type III polyketide synthases in Aspergillus oryzae. Biochem. Biophys. Res. Commun. 2005, 331, 253-260. [CrossRef] [PubMed]

20. Funa, N.; Awakawa, T.; Horinouchi, S. Pentaketide resorcylic acid synthesis by type III polyketide synthase from Neurospora crassa. J. Biol. Chem. 2007, 282, 14476-14481. [CrossRef] [PubMed]

21. Li, J.; Luo, Y.; Lee, J.K.; Zhao, H. Cloning and characterization of a type III polyketide synthase from Aspergillus niger. Bioorg. Med. Chem. Lett. 2011, 21, 6085-6089. [CrossRef] [PubMed]

22. Schuz, R.; Heller, W.; Hahlbrock, K. Substrate specificity of chalcone synthase from petroselinum hortense. J. Biol. Chem. 1983, 258, 6730-6734. [PubMed]

23. Ferrer, J.L.; Jez, J.M.; Bowman, M.E.; Dixon, R.A.; Noel, J.P. Structure of chalcone synthase and the molecular basis of plant polyketide biosynthesis. Nat. Struct. Biol. 1999, 6, 775-784. [PubMed]

24. Austin, M.B.; Noel, J.P. The chalcone synthase superfamily of type III polyketide synthases. Nat. Prod. Rep. 2003, 20, 79-110. [CrossRef] [PubMed]

25. Jez, J.M.; Ferrer, J.L.; Bowman, M.E.; Dixon, R.A.; Noel, J.P. Dissection of malonyl-coenzyme a decarboxylation from polyketide formation in the reaction mechanism of a plant polyketide synthase. Biochemistry 2000, 39, 890-902. [CrossRef] [PubMed]

26. Austin, M.B.; Bowman, M.E.; Ferrer, J.L.; Schroder, J.; Noel, J.P. An aldol switch discovered in stilbene synthases mediates cyclization specificity of type III polyketide synthases. Chem. Biol. 2004, 11, 1179-1194. [CrossRef] [PubMed]

27. Abe, I.; Morita, H. Structure and function of the chalcone synthase superfamily of plant type III polyketide synthases. Nat. Prod. Rep. 2010, 27, 809-838. [CrossRef] [PubMed]

28. Go, M.K.; Wongsantichon, J.; Cheung, V.W.N.; Chow, J.Y.; Robinson, R.C.; Yew, W.S. Synthetic polyketide enzymology: Platform for biosynthesis of antimicrobial polyketides. ACS Catal. 2015, 5, 4033-4042. [CrossRef]

29. Tropf, S.; Lanz, T.; Rensing, S.A.; Schroder, J.; Schroder, G. Evidence that stilbene synthases have developed from chalcone synthases several times in the course of evolution. J. Mol. Evol. 1994, 38, 610-618. [CrossRef] [PubMed]

30. Yamaguchi, T.; Kurosaki, F.; Suh, D.Y.; Sankawa, U.; Nishioka, M.; Akiyama, T.; Shibuya, M.; Ebizuka, Y. Cross-reaction of chalcone synthase and stilbene synthase overexpressed in Escherichia coli. FEBS Lett. 1999, 460, 457-461. [CrossRef]

31. Shomura, Y.; Torayama, I.; Suh, D.Y.; Xiang, T.; Kita, A.; Sankawa, U.; Miki, K. Crystal structure of stilbene synthase from Arachis hypogaea. Proteins 2005, 60, 803-806. [CrossRef] [PubMed]

32. Harris, T.M.; Carney, R.L. Biogenetically modeled synthesis of $\beta$-resorcylic acids. J. Am. Chem. Soc. 1966, 88, 2053-2054. [CrossRef]

33. Helariutta, Y.; Elomaa, P.; Kotilainen, M.; Griesbach, R.J.; Schroder, J.; Teeri, T.H. Chalcone synthase-like genes active during corolla development are differentially expressed and encode enzymes with different catalytic properties in Gerbera hybrida (asteraceae). Plant Mol. Biol. 1995, 28, 47-60. [CrossRef] [PubMed]

34. Eckermann, S.; Schroder, G.; Schmidt, J.; Strack, D.; Edrada, R.A.; Helariutta, Y.; Elomaa, P.; Kotilainen, M.; Kilpelainen, I.; Proksch, P.; et al. New pathway to polyketides in plants. Nature 1998, 396, 387-390. [CrossRef]

35. Jez, J.M.; Austin, M.B.; Ferrer, J.L.; Bowman, M.E.; Schroder, J.; Noel, J.P. Structural control of polyketide formation in plant-specific polyketide synthases. Chem. Biol. 2000, 7, 919-930. [CrossRef]

36. Abe, I.; Utsumi, Y.; Oguro, S.; Noguchi, H. The first plant type III polyketide synthase that catalyzes formation of aromatic heptaketide. FEBS Lett. 2004, 562, 171-176. [CrossRef]

37. Abe, I.; Watanabe, T.; Lou, W.; Noguchi, H. Active site residues governing substrate selectivity and polyketide chain length in aloesone synthase. FEBS J. 2006, 273, 208-218. [CrossRef] [PubMed] 
38. Morita, H.; Kondo, S.; Oguro, S.; Noguchi, H.; Sugio, S.; Abe, I.; Kohno, T. Structural insight into chain-length control and product specificity of pentaketide chromone synthase from Aloe arborescens. Chem. Biol. 2007, 14, 359-369. [CrossRef] [PubMed]

39. Mizuuchi, Y.; Shi, S.-P.; Wanibuchi, K.; Kojima, A.; Morita, H.; Noguchi, H.; Abe, I. Novel type III polyketide synthases from Aloe arborescens. FEBS J. 2009, 276, 2391-2401. [CrossRef] [PubMed]

40. Abe, I.; Oguro, S.; Utsumi, Y.; Sano, Y.; Noguchi, H. Engineered biosynthesis of plant polyketides: Chain length control in an octaketide-producing plant type III polyketide synthase. J. Am. Chem. Soc. 2005, 127, 12709-12716. [CrossRef] [PubMed]

41. Akiyama, T.; Shibuya, M.; Liu, H.M.; Ebizuka, Y. P-coumaroyltriacetic acid synthase, a new homologue of chalcone synthase, from Hydrangea macrophylla var. thunbergii. Eur. J. Biochem. 1999, 263, 834-839. [CrossRef] [PubMed]

42. Eckermann, C.; Schroder, G.; Eckermann, S.; Strack, D.; Schmidt, J.; Schneider, B.; Schroder, J. Stilbenecarboxylate biosynthesis: A new function in the family of chalcone synthase-related proteins. Phytochemistry 2003, 62, 271-286. [CrossRef]

43. Preisig-Muller, R.; Gnau, P.; Kindl, H. The inducible 9,10-dihydrophenanthrene pathway: Characterization and expression of bibenzyl synthase and s-adenosylhomocysteine hydrolase. Arch. Biochem. Biophys. 1995, 317, 201-207. [CrossRef] [PubMed]

44. Abe, I.; Takahashi, H.; Morita, H.; Noguchi, H. Benzalacetone synthase. A novel polyketide synthase that plays a crucial role in the biosynthesis of phenylbutanones in Rheum palmatum. Eur. J. Biochem. 2001, 268, 3354-3359. [CrossRef] [PubMed]

45. Morita, H.; Shimokawa, Y.; Tanio, M.; Kato, R.; Noguchi, H.; Sugio, S.; Kohno, T.; Abe, I. A structure-based mechanism for benzalacetone synthase from Rheum palmatum. Proc. Natl. Acad. Sci. USA 2010, 107, 669-673. [CrossRef] [PubMed]

46. Shimokawa, Y.; Morita, H.; Abe, I. Structure-based engineering of benzalacetone synthase. Bioorg. Med. Chem. Lett. 2010, 20, 5099-5103. [CrossRef] [PubMed]

47. Lu, H.; Yang, M.; Liu, C.; Lu, P.; Cang, H.; Ma, L. Protein preparation, crystallization and preliminary X-ray analysis of Polygonum cuspidatum bifunctional chalcone synthase/benzalacetone synthase. Acta Crystallogr. Sec. F Struct. Biol. Cryst. Commun. 2013, 69, 871-875. [CrossRef] [PubMed]

48. Katsuyama, Y.; Kita, T.; Funa, N.; Horinouchi, S. Curcuminoid biosynthesis by two type III polyketide synthases in the herb curcuma longa. J. Biol. Chem. 2009, 284, 11160-11170. [CrossRef] [PubMed]

49. Morita, H.; Wanibuchi, K.; Nii, H.; Kato, R.; Sugio, S.; Abe, I. Structural basis for the one-pot formation of the diarylheptanoid scaffold by curcuminoid synthase from oryza sativa. Proc. Natl. Acad. Sci. USA 2010, 107, 19778-19783. [CrossRef] [PubMed]

50. Beerhues, L.; Liu, B. Biosynthesis of biphenyls and benzophenones-evolution of benzoic acid-specific type III polyketide synthases in plants. Phytochemistry 2009, 70, 1719-1727. [CrossRef] [PubMed]

51. Liu, B.; Falkenstein-Paul, H.; Schmidt, W.; Beerhues, L. Benzophenone synthase and chalcone synthase from Hypericum androsaemum cell cultures: Cdna cloning, functional expression, and site-directed mutagenesis of two polyketide synthases. Plant J. 2003, 34, 847-855. [CrossRef] [PubMed]

52. Nualkaew, N.; Morita, H.; Shimokawa, Y.; Kinjo, K.; Kushiro, T.; De-Eknamkul, W.; Ebizuka, Y.; Abe, I. Benzophenone synthase from garcinia mangostana 1. Pericarps. Phytochemistry 2012, 77, 60-69. [CrossRef] [PubMed]

53. Beerhues, L. Benzophenone synthase from cultured cells of Centaurium erythraea. FEBS Lett. 1996, 383, 264-266. [CrossRef]

54. Liu, B.; Raeth, T.; Beuerle, T.; Beerhues, L. Biphenyl synthase, a novel type III polyketide synthase. Planta 2007, 225, 1495-1503. [CrossRef] [PubMed]

55. Baumert, A.; Maier, W.; Groger, D.; Deutzmann, R. Purification and properties of acridone synthase from cell suspension cultures of Ruta graveolens L. Z. Naturforsch. C 1994, 49, 26-32. [PubMed]

56. Springob, K.; Lukacin, R.; Ernwein, C.; Groning, I.; Matern, U. Specifities of functionally expressed chalcone and acridone synthases from Ruta graveolens. Eur. J. Biochem. 2000, 267, 6552-6559. [CrossRef] [PubMed]

57. Resmi, M.S.; Verma, P.; Gokhale, R.S.; Soniya, E.V. Identification and characterization of a type III polyketide synthase involved in quinolone alkaloid biosynthesis from Aegle marmelos correa. J. Biol. Chem. 2013, 288, 7271-7281. [CrossRef] [PubMed] 
58. Lukacin, R.; Schreiner, S.; Matern, U. Transformation of acridone synthase to chalcone synthase. FEBS Lett. 2001, 508, 413-417. [CrossRef]

59. Mori, T.; Shimokawa, Y.; Matsui, T.; Kinjo, K.; Kato, R.; Noguchi, H.; Sugio, S.; Morita, H.; Abe, I. Cloning and structure-function analyses of quinolone- and acridone-producing novel type III polyketide synthases from Citrus microcarpa. J. Biol. Chem. 2013, 288, 28845-28858. [CrossRef] [PubMed]

60. Meslet-Cladiere, L.; Delage, L.; Leroux, C.J.; Goulitquer, S.; Leblanc, C.; Creis, E.; Gall, E.A.; Stiger-Pouvreau, V.; Czjzek, M.; Potin, P. Structure/function analysis of a type III polyketide synthase in the brown alga ectocarpus siliculosus reveals a biochemical pathway in phlorotannin monomer biosynthesis. Plant Cell 2013, 25, 3089-3103. [CrossRef] [PubMed]

61. Miyanaga, A.; Horinouchi, S. Enzymatic synthesis of bis-5-alkylresorcinols by resorcinol-producing type III polyketide synthases. J. Antibiot. 2009, 62, 371-376. [CrossRef] [PubMed]

62. Kim, S.S.; Grienenberger, E.; Lallemand, B.; Colpitts, C.C.; Kim, S.Y.; Souza Cde, A.; Geoffroy, P.; Heintz, D.; Krahn, D.; Kaiser, M.; et al. LAP6/POLYKETIDE SYNTHASE A and LAP5/POLYKETIDE SYNTHASE B encode hydroxyalkyl $\alpha$-pyrone synthases required for pollen development and sporopollenin biosynthesis in Arabidopsis thaliana. Plant Cell 2010, 22, 4045-4066. [CrossRef] [PubMed]

63. Colpitts, C.C.; Kim, S.S.; Posehn, S.E.; Jepson, C.; Kim, S.Y.; Wiedemann, G.; Reski, R.; Wee, A.G.; Douglas, C.J.; Suh, D.Y. Ppascl, a moss ortholog of anther-specific chalcone synthase-like enzymes, is a hydroxyalkylpyrone synthase involved in an evolutionarily conserved sporopollenin biosynthesis pathway. New Phytol. 2011, 192, 855-868. [CrossRef] [PubMed]

64. Funa, N.; Ohnishi, Y.; Ebizuka, Y.; Horinouchi, S. Properties and substrate specificity of rppa, a chalcone synthase-related polyketide synthase in Streptomyces griseus. J. Biol. Chem. 2002, 277, 4628-4635. [CrossRef] [PubMed]

65. Izumikawa, M.; Shipley, P.R.; Hopke, J.N.; O’Hare, T.; Xiang, L.; Noel, J.P.; Moore, B.S. Expression and characterization of the type III polyketide synthase 1,3,6,8-tetrahydroxynaphthalene synthase from Streptomyces coelicolor A3(2). J. Ind. Microbiol. Biotechnol. 2003, 30, 510-515. [CrossRef] [PubMed]

66. Austin, M.B.; Izumikawa, M.; Bowman, M.E.; Udwary, D.W.; Ferrer, J.L.; Moore, B.S.; Noel, J.P. Crystal structure of a bacterial type III polyketide synthase and enzymatic control of reactive polyketide intermediates. J. Biol. Chem. 2004, 279, 45162-45174. [CrossRef] [PubMed]

67. Achkar, J.; Xian, M.; Zhao, H.; Frost, J.W. Biosynthesis of phloroglucinol. J. Am. Chem. Soc. 2005, 127, 5332-5333. [CrossRef] [PubMed]

68. Zha, W.; Rubin-Pitel, S.B.; Zhao, H. Characterization of the substrate specificity of phld, a type III polyketide synthase from Pseudomonas fluorescens. J. Biol. Chem. 2006, 281, 32036-32047. [CrossRef] [PubMed]

69. Tseng, C.C.; McLoughlin, S.M.; Kelleher, N.L.; Walsh, C.T. Role of the active site cysteine of dpga, a bacterial type III polyketide synthase. Biochemistry 2004, 43, 970-980. [CrossRef] [PubMed]

70. Wu, H.-C.; Li, Y.-S.; Liu, Y.-C.; Lyu, S.-Y.; Wu, C.-J.; Li, T.-L. Chain elongation and cyclization in type III pks dpga. ChemBioChem Eur. J. Chem. Biol. 2012, 13, 862-871. [CrossRef] [PubMed]

71. Saxena, P.; Yadav, G.; Mohanty, D.; Gokhale, R.S. A new family of type III polyketide synthases in Mycobacterium tuberculosis. J. Biol. Chem. 2003, 278, 44780-44790. [CrossRef] [PubMed]

72. Sankaranarayanan, R.; Saxena, P.; Marathe, U.B.; Gokhale, R.S.; Shanmugam, V.M.; Rukmini, R. A novel tunnel in mycobacterial type III polyketide synthase reveals the structural basis for generating diverse metabolites. Nat. Struct. Mol. Biol. 2004, 11, 894-900. [CrossRef] [PubMed]

73. Gokulan, K.; O’Leary, S.E.; Russell, W.K.; Russell, D.H.; Lalgondar, M.; Begley, T.P.; Ioerger, T.R.; Sacchettini, J.C. Crystal structure of Mycobacterium tuberculosis polyketide synthase 11 (pks11) reveals intermediates in the synthesis of methyl-branched alkylpyrones. J. Biol. Chem. 2013, 288, 16484-16494. [CrossRef] [PubMed]

74. Song, L.; Barona-Gomez, F.; Corre, C.; Xiang, L.; Udwary, D.W.; Austin, M.B.; Noel, J.P.; Moore, B.S.; Challis, G.L. Type III polyketide synthase beta-ketoacyl-acp starter unit and ethylmalonyl-coa extender unit selectivity discovered by Streptomyces coelicolor genome mining. J. Am. Chem. Soc. 2006, 128, 14754-14755. [CrossRef] [PubMed]

75. Gruschow, S.; Buchholz, T.J.; Seufert, W.; Dordick, J.S.; Sherman, D.H. Substrate profile analysis and acp-mediated acyl transfer in Streptomyces coelicolor type III polyketide synthases. ChemBioChem Eur. J. Chem. Biol. 2007, 8, 863-868. [CrossRef] [PubMed] 
76. Chemler, J.A.; Buchholz, T.J.; Geders, T.W.; Akey, D.L.; Rath, C.M.; Chlipala, G.E.; Smith, J.L.; Sherman, D.H. Biochemical and structural characterization of germicidin synthase: Analysis of a type III polyketide synthase that employs acyl-acp as a starter unit donor. J. Am. Chem. Soc. 2012, 134, 7359-7366. [CrossRef] [PubMed]

77. Jeya, M.; Kim, T.S.; Tiwari, M.K.; Li, J.; Zhao, H.; Lee, J.K. A type III polyketide synthase from Rhizobium etli condenses malonyl coas to a heptaketide pyrone with unusually high catalytic efficiency. Mol. BioSyst. 2012, 8, 3103-3106. [CrossRef] [PubMed]

78. Funa, N.; Ozawa, H.; Hirata, A.; Horinouchi, S. Phenolic lipid synthesis by type III polyketide synthases is essential for cyst formation in Azotobacter vinelandii. Proc. Natl. Acad. Sci. USA 2006, 103, 6356-6361. [CrossRef] [PubMed]

79. Miyanaga, A.; Funa, N.; Awakawa, T.; Horinouchi, S. Direct transfer of starter substrates from type I fatty acid synthase to type III polyketide synthases in phenolic lipid synthesis. Proc. Natl. Acad. Sci. USA 2008, 105, 871-876. [CrossRef] [PubMed]

80. Satou, R.; Miyanaga, A.; Ozawa, H.; Funa, N.; Katsuyama, Y.; Miyazono, K.; Tanokura, M.; Ohnishi, Y.; Horinouchi, S. Structural basis for cyclization specificity of two azotobacter type III polyketide synthases: A single amino acid substitution reverses their cyclization specificity. J. Biol. Chem. 2013, 288, 34146-34157. [CrossRef] [PubMed]

81. Awakawa, T.; Sugai, Y.; Otsutomo, K.; Ren, S.; Masuda, S.; Katsuyama, Y.; Horinouchi, S.; Ohnishi, Y. 4-hydroxy-3-methyl-6-(1-methyl-2-oxoalkyl)pyran-2-one synthesis by a type III polyketide synthase from Rhodospirillum centenum. ChemBioChem Eur. J. Chem. Biol. 2013, 14, 1006-1013. [CrossRef] [PubMed]

82. Muggia, L.; Grube, M. Type III polyketide synthases in lichen mycobionts. Fungal Biol. 2010, 114, $379-385$. [PubMed]

83. Goyal, A.; Saxena, P.; Rahman, A.; Singh, P.K.; Kasbekar, D.P.; Gokhale, R.S.; Sankaranarayanan, R. Structural insights into biosynthesis of resorcinolic lipids by a type III polyketide synthase in Neurospora crassa. J. Struct. Biol. 2008, 162, 411-421. [CrossRef] [PubMed]

84. Rubin-Pitel, S.B.; Zhang, H.; Vu, T.; Brunzelle, J.S.; Zhao, H.; Nair, S.K. Distinct structural elements dictate the specificity of the type III pentaketide synthase from Neurospora crassa. Chem. Biol. 2008, 15, 1079-1090. [CrossRef] [PubMed]

85. Hashimoto, M.; Nonaka, T.; Fujii, I. Fungal type III polyketide synthases. Nat. Prod. Rep. 2014, 31, $1306-1317$. [CrossRef] [PubMed]

86. Hashimoto, M.; Koen, T.; Takahashi, H.; Suda, C.; Kitamoto, K.; Fujii, I. Aspergillus oryzae csyb catalyzes the condensation of two beta-ketoacyl-coas to form 3-acetyl-4-hydroxy-6-alkyl-alpha-pyrone. J. Biol. Chem. 2014, 289, 19976-19984. [CrossRef] [PubMed]

87. Jeya, M.; Kim, T.S.; Tiwari, M.K.; Li, J.; Zhao, H.; Lee, J.K. The Botrytis cinerea type III polyketide synthase shows unprecedented high catalytic efficiency toward long chain acyl-coas. Mol. BioSyst. 2012, 8, 2864-2867. [CrossRef] [PubMed]

88. Yokoigawa, J.; Morimoto, K.; Shiono, Y.; Uesugi, S.; Kimura, K.I.; Kataoka, T. Allantopyrone a, an $\alpha$-pyrone metabolite from an endophytic fungus, inhibits the tumor necrosis factor $\alpha$-induced nuclear factor kappab signaling pathway. J. Antibiot. 2015, 68, 71-75. [CrossRef] [PubMed]

89. Zhou, X.M.; Zheng, C.J.; Song, X.P.; Han, C.R.; Chen, W.H.; Chen, G.Y. Antibacterial $\alpha$-pyrone derivatives from a mangrove-derived fungus Stemphylium sp. 33231 from the south China sea. J. Antibiot. 2014, 67, 401-403. [CrossRef] [PubMed]

90. Abe, I.; Takahashi, Y.; Lou, W.; Noguchi, H. Enzymatic formation of unnatural novel polyketides from alternate starter and nonphysiological extension substrate by chalcone synthase. Org. Lett. 2003, 5, 1277-1280. [CrossRef] [PubMed]

91. Samappito, S.; Page, J.; Schmidt, J.; De-Eknamkul, W.; Kutchan, T.M. Molecular characterization of root-specific chalcone synthases from Cassia alata. Planta 2002, 216, 64-71. [CrossRef] [PubMed]

92. Morita, H.; Noguchi, H.; Schroder, J.; Abe, I. Novel polyketides synthesized with a higher plant stilbene synthase. Eur. J. Biochem. 2001, 268, 3759-3766. [CrossRef] [PubMed]

93. Li, T.L.; Spiteller, D.; Spencer, J.B. Identification of a pentaketide stilbene produced by a type III polyketide synthase from pinus sylvestris and characterisation of free coenzyme a intermediates. ChemBioChem Eur. J. Chem. Biol. 2009, 10, 896-901. [CrossRef] [PubMed] 
94. Shi, S.-P.; Wanibuchi, K.; Morita, H.; Endo, K.; Noguchi, H.; Abe, I. Enzymatic formation of unnatural novel chalcone, stilbene, and benzophenone scaffolds by plant type III polyketide synthase. Org. Lett. 2009, 11, 551-554. [CrossRef] [PubMed]

95. Karppinen, K.; Hokkanen, J.; Mattila, S.; Neubauer, P.; Hohtola, A. Octaketide-producing type III polyketide synthase from hypericum perforatum is expressed in dark glands accumulating hypericins. FEBS J. 2008, 275, 4329-4342. [CrossRef] [PubMed]

96. Abe, I.; Abe, T.; Wanibuchi, K.; Noguchi, H. Enzymatic formation of quinolone alkaloids by a plant type III polyketide synthase. Org. Lett. 2006, 8, 6063-6065. [CrossRef] [PubMed]

97. Wakimoto, T.; Mori, T.; Morita, H.; Abe, I. Cytotoxic tetramic acid derivative produced by a plant type-III polyketide synthase. J. Am. Chem. Soc. 2011, 133, 4746-4749. [CrossRef] [PubMed]

98. Go, M.K.; Chow, J.Y.; Cheung, V.W.; Lim, Y.P.; Yew, W.S. Establishing a toolkit for precursor-directed polyketide biosynthesis: Exploring substrate promiscuities of acid-coa ligases. Biochemistry 2012, 51, 4568-4579. [CrossRef] [PubMed]

99. Klundt, T.; Bocola, M.; Lutge, M.; Beuerle, T.; Liu, B.; Beerhues, L. A single amino acid substitution converts benzophenone synthase into phenylpyrone synthase. J. Biol. Chem. 2009, 284, 30957-30964. [CrossRef] [PubMed]

100. Jez, J.M.; Bowman, M.E.; Noel, J.P. Expanding the biosynthetic repertoire of plant type III polyketide synthases by altering starter molecule specificity. Proc. Natl. Acad. Sci. USA 2002, 99, 5319-5324. [CrossRef] [PubMed]

101. Abe, I.; Watanabe, T.; Morita, H.; Kohno, T.; Noguchi, H. Engineered biosynthesis of plant polyketides-Manipulation of chalcone synthase. Org. Lett. 2006, 8, 499-502. [CrossRef] [PubMed]

102. Abe, I.; Morita, H.; Oguro, S.; Noma, H.; Wanibuchi, K.; Kawahara, N.; Goda, Y.; Noguchi, H.; Kohno, T. Structure-based engineering of a plant type III polyketide synthase: Formation of an unnatural nonaketide naphthopyrone. J. Am. Chem. Soc. 2007, 129, 5976-5980. [CrossRef] [PubMed]

103. Abe, I. Engineered biosynthesis of plant polyketides: Structure-based and precursor-directed approach. Top. Curr. Chem. 2010, 297, 45-66. [PubMed]

104. Wanibuchi, K.; Morita, H.; Noguchi, H.; Abe, I. Enzymatic formation of an aromatic dodecaketide by engineered plant polyketide synthase. Bioorg. Med. Chem. Lett. 2011, 21, 2083-2086. [CrossRef] [PubMed]

105. Morita, H.; Yamashita, M.; Shi, S.P.; Wakimoto, T.; Kondo, S.; Kato, R.; Sugio, S.; Kohno, T.; Abe, I. Synthesis of unnatural alkaloid scaffolds by exploiting plant polyketide synthase. Proc. Natl. Acad. Sci. USA 2011, 108, 13504-13509. [CrossRef] [PubMed]

106. Funa, N.; Ohnishi, Y.; Ebizuka, Y.; Horinouchi, S. Alteration of reaction and substrate specificity of a bacterial type III polyketide synthase by site-directed mutagenesis. Biochem. J. 2002, 367, 781-789. [CrossRef] [PubMed]

107. Li, S.; Gruschow, S.; Dordick, J.S.; Sherman, D.H. Molecular analysis of the role of tyrosine 224 in the active site of Streptomyces coelicolor rppa, a bacterial type III polyketide synthase. J. Biol. Chem. 2007, 282, 12765-12772. [CrossRef] [PubMed]

(c) 2016 by the authors; licensee MDPI, Basel, Switzerland. This article is an open access article distributed under the terms and conditions of the Creative Commons Attribution (CC-BY) license (http://creativecommons.org/licenses/by/4.0/). 